\title{
Noise and dissipation in magnetoelectronic nanostructures
}

\author{
Jørn Foros and Arne Brataas \\ Department of Physics, Norwegian University of Science and Technology, 7491 Trondheim, Norway \\ Gerrit E. W. Bauer \\ Kavli Institute of NanoScience, Delft University of Technology, 2628 CJ Delft, The Netherlands \\ Yaroslav Tserkovnyak \\ Department of Physics and Astronomy, University of California, Los Angeles, California 90095, USA \\ (Received 22 February 2009; revised manuscript received 12 May 2009; published 8 June 2009)
}

\begin{abstract}
The interplay between current and magnetization fluctuations and dissipation in layered-ferromagneticnormal-metal nanostructures is investigated. We use scattering theory and magnetoelectronic circuit theory to calculate charge and spin-current fluctuations. Via the spin-transfer torque, spin-current noise causes a significant enhancement of magnetization fluctuations. A special focus is on spin valves in which one of the ferromagnets is pinned. We find that the magnetization noise and damping are tensors that depend on the magnetic configuration. For symmetric spin valves in which both layers fluctuate, dynamic cross-talk between the layers becomes important, causing a possibly large difference in noise level between the parallel and antiparallel magnetic configurations. Due to giant magnetoresistance (GMR), the magnetization fluctuations in spin valves induce resistance noise, which is identified as a prominent source of electric noise at relatively high current densities. The resistance noise is shown to vary considerably with the magnetic configuration, partly due to the dependence of the angular GMR. The contribution from spin-current fluctuations to the resistance noise is shown to be significant. Resistance noise is an experimentally accessible quantity that can be measured to verify our results.
\end{abstract}

DOI: $10.1103 /$ PhysRevB.79.214407

PACS number(s): 72.70.+m, 72.25.Mk, 75.75.+a

\section{INTRODUCTION}

New functionalities can be realized by integrating ferromagnetic elements into electronic circuits and devices. The interplay between magnetism and electric currents in these structures is utilized by the giant magnetoresistance (GMR), the operating principle of the read heads in modern magnetic hard disk drives. Considerable progress has been made in improving magnetic random access memories. ${ }^{1}$ Efforts to further miniaturize and improve the performance of magnetoelectronic devices are ongoing in academic and corporate laboratories. Low power consumption and noise levels are essential. In spite of the technological relevance, a comprehensive understanding of coupled current and magnetization noise, and the related energy dissipation in nanoscale magnetoelectronic circuits is lacking.

From the early studies of Johnson ${ }^{2}$ and Nyquist, ${ }^{3}$ we know that the equilibrium voltage noise power in conductors is proportional to the electric resistance. This relation between the equilibrium noise and the out-of-equilibrium energy dissipation is a standard example of the fluctuationdissipation theorem (FDT) ${ }^{4,5}$ In recent years, important advances have been made in the understanding of electronic equilibrium (thermal) and nonequilibrium (shot) noises in mesoscopic conductors. ${ }^{6}$

The electron spin plays an important role in electrical noise phenomena in magnetic multilayers. In early theoretical studies ${ }^{7-12}$ of charge and spin-polarized current noise in such systems, magnetizations were assumed to be static. However, the magnetization itself fluctuates as well. Thermal fluctuations of the magnetization vector in isolated singledomain ferromagnets have been analyzed by Brown, ${ }^{13}$ who introduced a stochastic Langevin field acting on the magnetization to account for thermal agitation. His proof that this field's (white-noise) correlator is proportional to the magnetization damping (see below) is another manifestation of the FDT. ${ }^{14,15}$ The stochastic field can be introduced into the spatiotemporal equation of motion for the magnetization [Landau-Lifshitz-Gilbert (LLG) equation], affecting, e.g., current-driven magnetization dynamics and reversal. ${ }^{16-19}$

A moving magnetization vector in ferromagnets undergoes viscous damping that relaxes the magnetization toward the lowest (free-) energy configuration. This process is in practice well described by a phenomenological damping constant, introduced by Gilbert. ${ }^{20,21}$ Despite some progress, ${ }^{22-28}$ a rigorous quantitative understanding of the magnetic damping in transition-metal ferromagnets has not yet been achieved. The theory of the enhanced Gilbert damping in ferromagnets in good electrical contact with a conducting environment is in a better shape. The loss of angular momentum due to spin-current pumping into the environment agrees with the Gilbert phenomenology, ${ }^{29,30}$ and experiment and theory addressing the additional damping agree well with each other. ${ }^{30}$

The electronic and magnetic fluctuations in magnetoelectronic structures are intimately coupled to each other. ${ }^{31,32}$ For example, the magnetization noise in ferromagnetic films in good electric contact with normal metals has been predicted to increase due to spin-current fluctuations: the spin-current components polarized perpendicularly to the magnetization are absorbed at the interface, leading to a fluctuating spintransfer torque ${ }^{33-36}$ that induces additional magnetization noise. This noise is related to the excess Gilbert damping caused by the angular-momentum loss due to spin pumping, in accordance with the FDT. 
Here we investigate the interplay between (zerofrequency) current and magnetization noise in multilayers of alternating monodomain magnetic and nonmagnetic films. We take advantage of the FDT to relate the equilibrium electric (current and voltage) and magnetic (magnetization and field) noises to the corresponding dissipation of energy. We start by reviewing the noise in a single monodomain ferromagnet sandwiched by normal metals, including technical details that were omitted in Ref. 31. Both thermal equilibrium (Johnson-Nyquist) current noise and nonequilibrium shot noise are taken into account. Next, we consider spin valves, i.e., two ferromagnetic films separated by a normalmetal spacer. ${ }^{37}$ We consider both a symmetric structure in which both layers fluctuate, as well as an asymmetric one, in which one layer is assumed fixed. Magnetoelectronic circuit theory ${ }^{38-40}$ is used to calculate the charge and spin-current fluctuations. The resulting enhanced magnetization noise and Gilbert damping in principle are tensors that depend on the magnetic configuration. Our focus is on uniformly magnetized ferromagnets that can be described in the so-called macrospin approximation. Additional interesting effects occur in nonuniformly magnetized ferromagnets. ${ }^{41}$

Spin valves provide an opportunity to indirectly measure magnetization noise via resistance fluctuations, which are manifested by voltage noise for a current-biased system or current noise for a voltage-biased system. ${ }^{42,43}$ This offers an experimental test of our theory. We obtain analytical expressions for the magnetic contribution to the induced electric noise for different magnetic configurations. The noise is of potential importance for the performance of spin-valve read heads. ${ }^{43}$ For symmetric structures in which both layers fluctuate, dynamic cross-talk between the layers becomes important, causing a possibly large difference in noise level between the parallel (P) and antiparallel (AP) magnetic configurations. Our results for these spin valves include previously presented findings as a limiting case. ${ }^{37}$ After the completion of this work, ${ }^{44}$ it was shown that spin valves in equilibrium also exhibit colored voltage fluctuations caused by spin pumping of the moving magnetizations. ${ }^{45}$

The paper is organized as follows. We begin by reviewing the fluctuation-dissipation theorem, applied to magnetic systems. In Sec. III, the noise properties of a single ferromagnetic thin film sandwiched by normal metals is worked out in detail, emphasizing the relation of the noise to the damping. In Sec. IV, we consider current noise, magnetization noise, and magnetization damping in spin valves, and use the results to calculate the resistance noise induced by GMR. Section $\mathrm{V}$ concludes our paper.

\section{FLUCTUATION-DISSIPATION THEOREM}

The FDT relates the spontaneous time-dependent changes in an observable of a given system in thermal equilibrium to its linear response to an external perturbation that couples to that observable. For example, in an electric conductor the spontaneous fluctuations in the electric current are proportional to the dissipative (real) part of the conductivity, i.e., the response function to an applied electric field. ${ }^{2,3}$ Similarly, the equilibrium fluctuations of the magnetization vector in a ferromagnet are proportional to the dissipative part of the magnetic susceptibility, i.e., imaginary part of the response function to an applied magnetic field. In the following, we briefly recapitulate this FDT for magnetic systems.

Sufficiently below the Curie temperature, changes in the modulus of the magnetization are energetically costly and may be disregarded. For sufficiently small magnetic structures spin waves freeze out of the problem. Hence, a small ferromagnetic particle or thin film is well described in terms of a single magnetization vector $M_{s} \mathbf{m}$, where $M_{s}$ is the magnitude of the magnetization and $\mathbf{m}$ is a unit vector ("macrospin" model). The time-dependent equilibrium fluctuations of the magnetization are characterized by the autocorrelation function $\left\langle\delta m_{i}(t) \delta m_{j}\left(t^{\prime}\right)\right\rangle$, where $\delta m_{i}(t)=m_{i}(t)-\left\langle m_{i}(t)\right\rangle$ are transverse fluctuations. Here the brackets denote statistical averaging at equilibrium, and $i$ and $j$ denote Cartesian components perpendicular to the equilibrium/average magnetization direction. The classical FDT states that these fluctuations are related to the magnetic susceptibility:

$$
\left\langle\delta m_{i}(t) \delta m_{j}\left(t^{\prime}\right)\right\rangle=\frac{k_{B} T}{2 \pi M_{s} V} \int d \omega e^{-i \omega\left(t-t^{\prime}\right)} \frac{\chi_{i j}(\omega)-\chi_{j i}^{*}(\omega)}{i \omega},
$$

where $T$ is the temperature, $V$ is the volume of the ferromagnet, and $\chi_{i j}(\omega)$ is the $i j$ component of the transverse magnetic susceptibility at frequency $\omega$. The latter is the linear (causal) response function that describes the changes in the magnetization, $\Delta m_{i}(t)$, caused by an external driving field $\mathbf{H}^{(\mathrm{dr})}(t)$ :

$$
\Delta m_{i}(t)=\sum_{j} \int d t^{\prime} \chi_{i j}\left(t-t^{\prime}\right) H_{j}^{(\mathrm{dr})}\left(t^{\prime}\right)
$$

An alternative form of the FDT that turns out to be useful in the course of this paper can be derived by introducing a stochastic magnetic field $\mathbf{h}^{(0)}(t)$ with zero mean. This field effectively represents the coupling of the magnetization to the dissipative degrees of freedom, and is viewed as the cause of the thermal fluctuations $\delta \mathbf{m}(t)$. The microscopic origin of $\mathbf{h}^{(0)}(t)$ does not concern us here but it might, e.g., represent thermally excited phonons that deform the crystal anisotropy fields. From Eq. (2) it follows that $\delta m_{i}(\omega)$ $=\sum_{j} \chi_{i j}(\omega) h_{j}^{(0)}(\omega)$ in frequency domain. Inverting this relation, the correlator of the stochastic field has to obey the relation

$$
\left\langle h_{i}^{(0)}(t) h_{j}^{(0)}\left(t^{\prime}\right)\right\rangle=\frac{k_{B} T}{2 \pi M_{S} V} \int d \omega e^{-i \omega\left(t-t^{\prime}\right)} \frac{\left[\chi_{j i}^{-1}(\omega)\right]^{*}-\chi_{i j}^{-1}(\omega)}{i \omega},
$$

where $\chi_{i j}^{-1}(\omega)$ is the $i j$ component of the inverted (Fouriertransformed) susceptibility.

\section{SINGLE FERROMAGNET}

The magnetization dynamics of an isolated single-domain ferromagnet is well described by the LLG equation ${ }^{20,46}$ 


$$
\frac{d \mathbf{m}}{d t}=-\gamma_{0} \mathbf{m} \times \mathbf{H}_{\mathrm{eff}}+\alpha_{0} \mathbf{m} \times \frac{d \mathbf{m}}{d t},
$$

where $\gamma_{0}$ is the gyromagnetic ratio, $\mathbf{H}_{\mathrm{eff}}$ is the effective magnetic field, and $\alpha_{0}$ is the Gilbert damping constant. The effective field has contributions due to crystal and form anisotropies, as well as externally applied magnetic fields. By linearizing this LLG equation we can evaluate the magnetic susceptibility and the equilibrium magnetization noise. The average equilibrium direction of the magnetization is aligned with $\mathbf{H}_{\mathrm{eff}}$ to minimize the energy: $\mathbf{m}_{0}=\mathbf{H}_{\mathrm{eff}} /\left|\mathbf{H}_{\mathrm{eff}}\right|$. A weak external driving field is included by substituting $\mathbf{H}_{\mathrm{eff}}$ $\rightarrow \mathbf{H}_{\text {eff }}+\mathbf{H}^{(\mathrm{dr})}(t)$. In the present model only the component of $\mathbf{H}^{(\mathrm{dr})}$ transverse to the magnetization will solicit a response $\mathbf{m}(t) \approx \mathbf{m}_{0}+\Delta \mathbf{m}(t)$ of the magnetization. Here $\Delta \mathbf{m}(t)$ is normal to $\mathbf{m}_{0}$. To lowest order in $\Delta \mathbf{m}(t)$, the LLG equation gives the inverse susceptibility tensor matrix,

$$
\chi^{-1}=\frac{1}{\gamma_{0}}\left[\begin{array}{cc}
\gamma_{0}\left|\mathbf{H}_{\mathrm{eff}}\right|-i \omega \alpha_{0} & i \omega \\
-i \omega & \gamma_{0}\left|\mathbf{H}_{\mathrm{eff}}\right|-i \omega \alpha_{0}
\end{array}\right],
$$

in the plane normal to $\mathbf{m}_{0}$. This expression for transverse susceptibility (5) assumes that the effective magnetic field is a constant which is often not the case. However, a dependence of the effective field $\mathbf{H}_{\text {eff }}$ on $\mathbf{m}$ does not affect the intrinsic noise properties that we will now discuss and we will relax this assumption later in our paper.

The magnetization noise follows from substituting Eq. (5) into Eq. (1). The correlator of the stochastic field is obtained from Eqs. (3) and (5) and does not depend on the effective field: ${ }^{13}$

$$
\left\langle h_{i}^{(0)}(t) h_{j}^{(0)}\left(t^{\prime}\right)\right\rangle=2 k_{B} T \frac{\alpha_{0}}{\gamma_{0} M_{s} V} \delta_{i j} \delta\left(t-t^{\prime}\right) .
$$

The relation between the equilibrium magnetization fluctuations and the dissipation in the form of the Gilbert damping is evident.

Up to now we considered a ferromagnet isolated from the outside world. Its dynamics is altered by embedding into a conducting environment. ${ }^{29} \mathrm{~A}$ ferromagnet with timedependent magnetization "pumps" an angular-momentum (spin) current,

$$
\mathbf{I}_{s}^{\text {pump }}=\frac{\hbar}{4 \pi}\left(\operatorname{Re} g^{\uparrow \downarrow} \mathbf{m} \times \frac{d \mathbf{m}}{d t}+\operatorname{Im} g^{\uparrow \downarrow} \frac{d \mathbf{m}}{d t}\right),
$$

into an adjacent conductor. Here $g^{\uparrow \downarrow}$ is the dimensionless transverse spin ("mixing") conductance that depends on the interface transparency between ferromagnet and proximate metal. ${ }^{38-40}$ When the spin current is efficiently dissipated in the conductor, thus not building up a spin accumulation close to the interface, the loss of angular momentum corresponds to an extra torque $\gamma \mathbf{I}_{s}^{\text {pump }} /\left(M_{s} V\right)$ on the right-hand side of Eq. (4). This is equivalent to an increased Gilbert damping and a modified gyromagnetic ratio: ${ }^{29}$

$$
\frac{1}{\gamma_{0}} \rightarrow \frac{1}{\gamma}=\frac{1}{\gamma_{0}}\left(1-\frac{\gamma_{0} \hbar \operatorname{Im} g^{\uparrow \downarrow}}{4 \pi M_{s} V}\right),
$$

$$
\alpha_{0} \rightarrow \alpha=\frac{\gamma}{\gamma_{0}}\left(\alpha_{0}+\frac{\gamma_{0} \hbar \operatorname{Re} g^{\uparrow \downarrow}}{4 \pi M_{s} V}\right) .
$$

In the strong-coupling limit (intermetallic interfaces), $\operatorname{Im} g^{\uparrow \downarrow} \ll \operatorname{Re} g^{\uparrow \downarrow}$, and we are allowed to disregard the difference between $\gamma$ and $\gamma_{0}$.

Another term which modifies the magnetization dynamics is the so-called spin-transfer torque. ${ }^{33,34}$ It is also proportional to the spin-mixing conductance introduced above ${ }^{38-40}$ and represented by adding $-\gamma_{0} \mathbf{I}_{s, \text { abs }} /\left(M_{s} V\right)$ to the right-hand side of Eq. (4). Here $\mathbf{I}_{s, \text { abs }}$ is the spin-polarized current transversely polarized to the magnetization, which is absorbed by the ferromagnet on an atomic length scale, thereby transferring its angular momentum to the magnetization. Spin pumping and spin-transfer torque are related by an Onsager reciprocity relation. ${ }^{47}$

Recently we have shown ${ }^{31}$ that the magnetization noise in magnetoelectronic nanostructures can be considerably increased as compared to an isolated ferromagnet. At elevated temperatures, thermal fluctuations in the spin current exert a fluctuating torque on the magnetization, increasing the noise. For a ferromagnet sandwiched by normal metals, the enhancement of the noise is described by a stochastic field $\mathbf{h}^{(\text {th) }}(t)$ similar to the intrinsic field $\mathbf{h}^{(0)}(t)$. Its correlation function reads ${ }^{31}$

$$
\left\langle h_{i}^{(\mathrm{th})}(t) h_{j}^{(\mathrm{th})}\left(t^{\prime}\right)\right\rangle=2 k_{B} T \frac{\alpha^{\prime}}{\gamma M_{s} V} \delta_{i j} \delta\left(t-t^{\prime}\right),
$$

where

$$
\alpha^{\prime}=\frac{\gamma \hbar \operatorname{Re} g^{\uparrow \downarrow}}{4 \pi M_{s} V}
$$

is the enhancement of the Gilbert damping due to spin pumping [see Eq. (9)]. Assuming that $\mathbf{h}^{(0)}(t)$ and $\mathbf{h}^{(\text {th })}(t)$ are statistically independent, the total magnetization noise is thus given by $\mathbf{h}(t)=\mathbf{h}^{(0)}(t)+\mathbf{h}^{(\text {th) }}(t)$. We know that the total damping is determined by $\alpha=\alpha_{0}+\alpha^{\prime}$, and from Eqs. (6) and (10) we see that the total noise is related to the total damping, in agreement with the FDT. Hence, the thermal spin-current noise is the stochastic process related to the enhanced dissipation of energy by spin pumping. By calculating the noise power we also know the damping and vice versa. In thin ferromagnetic films, $\alpha^{\prime}$ can be of the same order or even larger than $\alpha_{0} .{ }^{30}$ In the following subsections we will give a detailed derivation of Eq. (10). We also evaluate the shotnoise contribution to the magnetization noise, which is important at low temperatures. ${ }^{31}$ We note here that Eq. (10) may be found also by direct application of Eq. (3) to the LLG equation with spin pumping included.

\section{A. Scattering theory}

We study a thin ferromagnetic film connected to two normal reservoirs, as shown in Fig. 1. The reservoirs are perfect spin sinks and the ferromagnet is taken to be thicker than the magnetic coherence length $\lambda_{c}=\pi /\left(k_{\uparrow}-k_{\downarrow}\right)$, where $k_{\uparrow(\downarrow)}$ are spin-dependent Fermi momenta. For transition metals, $\lambda_{c}$ is of the order of monolayers. The normal metals are character- 


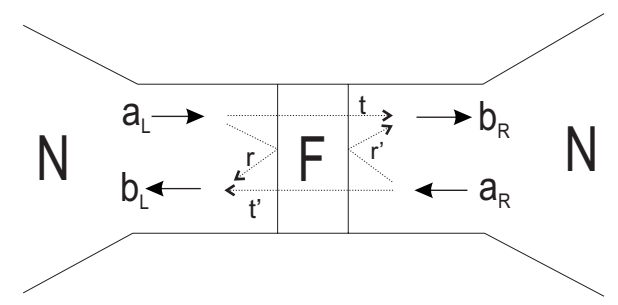

FIG. 1. A thin ferromagnetic (F) film is sandwiched by normal metals $(\mathrm{N})$. The current fluctuations in the system are evaluated in terms of transmission probabilities for the electron states, with the aid of second-quantized annihilation and creation operators. The operators shown in the figure are annihilation operators, with the $a$ operators annihilating electrons moving toward the ferromagnet, and the $b$ operators annihilating electrons moving away from the ferromagnet. Also shown are the reflection and transmission matrices $r, r^{\prime}, t, t^{\prime}$ [see Eq. (14)], for simplicity without spin indices.

ized by Fermi-Dirac distribution functions $f_{L}$ and $f_{R}$ with chemical potentials $\mu_{L}$ and $\mu_{R}$, where $L$ and $R$ refer to the left and right sides at a common temperature $T$. We use the Landauer-Büttiker (LB) scattering theory ${ }^{6}$ to evaluate the spin-current fluctuations, and the LLG equation to calculate the resulting magnetization noise.

In the LB approach electron transport is expressed in terms of transmission probabilities between the electron states on different sides of a scattering region. Here we interpret the ferromagnetic film as a scatterer that limits the propagation of electrons between the normal reservoirs. The scattering properties of the ferromagnet and the bias between the reservoirs determine the transport properties of the system. The transport channels in the leads are modeled as ideal electron waveguides in which the transverse and longitudinal motions are separable. The transport channels at a given energy $E$ are then labeled by the discrete mode index for the quantized transverse motion, by which the continuous-wave vector for the longitudinal motion is fixed. The LB formalism $^{6,48}$ generalized to describe spin transport leads to the current operator,

$$
\begin{aligned}
\hat{I}_{A}^{\alpha \beta}(t)= & \frac{e}{h} \int d E d E^{\prime} e^{i\left(E-E^{\prime}\right) t / \hbar} \\
& \times\left[a_{A \beta}^{\dagger}(E) a_{A \alpha}\left(E^{\prime}\right)-b_{A \beta}^{\dagger}(E) b_{A \alpha}\left(E^{\prime}\right)\right],
\end{aligned}
$$

at time $t$ on side $A[=L$ (left) or $R$ (right)] of the ferromagnetic film. Here, $\alpha$ and $\beta$ denote components in $2 \times 2$ spin space. $a_{A \alpha}(E)$ and $b_{A \alpha}(E)$ are operators for all transport channels at energy $E$ that annihilate electrons with spin $\alpha$ in lead $A$ that move toward and away from the ferromagnet, respectively (see Fig. 1). The $a$ operators are related to the $b$ operators by the scattering properties of the ferromagnet:

$$
b_{A \alpha}(E)=\sum_{B \beta} s_{A B \alpha \beta}(E) a_{B \beta}(E),
$$

where $s_{A B \alpha \beta}$ is the scattering matrix for incoming electrons with spin $\beta$ in lead $B(=L$ or $R$ ) scattered to outgoing states in lead $A$ with spin $\alpha$. The summation is over $B=L, R$ and over spin $\beta=\uparrow, \downarrow$. A similar relation holds for the creation opera- tors. Current conservation implies that the scattering matrix is unitary. Suppressing spin indices for simplicity (see Fig. 1)

$$
\left(\begin{array}{l}
b_{L} \\
b_{R}
\end{array}\right)=\left(\begin{array}{ll}
r & t^{\prime} \\
t & r^{\prime}
\end{array}\right)\left(\begin{array}{l}
a_{L} \\
a_{R}
\end{array}\right),
$$

where $r=s_{L L}, r^{\prime}=s_{R R}, t=s_{R L}$, and $t^{\prime}=s_{L R}$. In the following we disregard spin-flip processes in the ferromagnet. Choosing the spin-quantization $z$ axis in the direction of the average magnetization, this implies that $s_{A B \alpha \beta}=s_{A B \alpha} \delta_{\alpha \beta}$.

The outgoing charge and spin currents are given, respectively, by $I_{c, A}(t)=\Sigma_{\alpha} \hat{I}_{A}^{\alpha \alpha}(t)$ and $\mathbf{I}_{s, A}(t)=-(\hbar / 2 e) \Sigma_{\alpha \beta} \hat{\boldsymbol{\sigma}}^{\alpha \beta} \hat{I}_{A}^{\beta \alpha}(t)$, where $\hat{\boldsymbol{\sigma}}=\left(\hat{\sigma}_{x}, \hat{\sigma}_{y}, \hat{\sigma}_{z}\right)$ is the vector of Pauli matrices. The expectation values for charge and spin currents are evaluated using the quantum statistical average $\left\langle a_{A m \alpha}^{\dagger}(E) a_{B n \beta}\left(E^{\prime}\right)\right\rangle$ $=\delta_{A B} \delta_{m n} \delta_{\alpha \beta} \delta\left(E-E^{\prime}\right) f_{A}(E)$ of the product of one creation and one annihilation operator, where $m$ and $n$ label the transport channels. The creation and annihilation operators obey the anticommutation relation

$$
\left\{a_{A m \alpha}^{\dagger}(E), a_{B n \beta}\left(E^{\prime}\right)\right\}=\delta_{A B} \delta_{m n} \delta_{\alpha \beta} \delta\left(E-E^{\prime}\right),
$$

whereas the anticommutators of two creation or two annihilation operators vanish. Similar relations hold for the $b$ operators. The average

$$
\begin{aligned}
\left\langle a_{A k \alpha}^{\dagger}\right. & \left.\left(E_{1}\right) a_{B l \beta}\left(E_{2}\right) a_{C m \gamma}^{\dagger}\left(E_{3}\right) a_{D n \delta}\left(E_{4}\right)\right\rangle \\
- & \left\langle a_{A k \alpha}^{\dagger}\left(E_{1}\right) a_{B l \beta}\left(E_{2}\right)\right\rangle\left\langle a_{C m \gamma}^{\dagger}\left(E_{3}\right) a_{D n \delta}\left(E_{4}\right)\right\rangle \\
= & \delta_{A D} \delta_{B C} \delta_{k n} \delta_{l m} \delta_{\alpha \delta} \delta_{\beta \gamma} \delta\left(E_{1}-E_{4}\right) \\
& \times \delta\left(E_{2}-E_{3}\right) f_{A}\left(E_{1}\right)\left[1-f_{B}\left(E_{2}\right)\right],
\end{aligned}
$$

where the subscripts $A, B, C, D$ denote leads, $k, l, m, n$ denote transport channels, and $\alpha, \beta, \gamma, \delta$ denote spins, is needed for the calculation of the current fluctuations. We also need the identity

$$
\sum_{C D} \operatorname{Tr}\left(s_{A C \alpha}^{\dagger} s_{A D \beta} s_{B D \beta}^{\dagger} s_{B C \alpha}\right)=\delta_{A B} M_{A},
$$

which follows from the unitarity of the scattering matrix. Here the trace is over the space of the transport channels, and $M_{A}$ is the number of transverse channels in lead $A$, all at a given energy.

The charge and spin-current correlation functions read

$$
S_{c, A B}\left(t-t^{\prime}\right)=\left\langle\delta I_{c, A}(t) \delta I_{c, B}\left(t^{\prime}\right)\right\rangle,
$$

and

$$
S_{i j, A B}\left(t-t^{\prime}\right)=\left\langle\delta I_{s_{i}, A}(t) \delta I_{s_{j}, B}\left(t^{\prime}\right)\right\rangle,
$$

where $\delta I_{c, A}(t)=I_{c, A}(t)-\left\langle I_{c, A}(t)\right\rangle$ denotes the deviation of the charge current from its average value in lead $A$ at time $t$, and $\delta I_{s_{i}, A}(t)$ is the deviation of the vector component $i$ ( $i=x, y$, or $z$ ) of the spin current. We are interested mainly in the lowfrequency noise, i.e., the time integrated value of the correlation functions:

$$
S_{c, A B}(\omega=0)=\int d\left(t-t^{\prime}\right) S_{c, A B}\left(t-t^{\prime}\right) .
$$

Two fundamentally different types of current noise have to be distinguished: thermal (equilibrium) noise and (nonequi- 
librium) shot noise. In general, the total noise is not simply a linear combination of both types. Nevertheless, it is convenient to treat the two noise sources independently, by separately investigating the noise of an unbiased system at finite temperatures in Sec. III B and the shot noise under an applied bias at zero temperature in Sec. III C.

\section{B. Thermal noise}

At equilibrium $f_{L}=f_{R}=f$, and the average current vanishes. However, at finite temperatures, the occupation numbers of the electron channels incident on the sample fluctuate in time and so does the current. Using Eqs. (12), (13), (16), and (17), and $f(1-f)=k_{B} T(-\partial f / \partial E)$, we recover the wellknown Johnson-Nyquist noise,

$$
S_{c, A A}^{(\text {th })}(\omega=0)=\frac{2 e^{2}}{h} k_{B} T\left(g^{\uparrow}+g^{\downarrow}\right),
$$

in the zero-frequency limit. Here $g^{\alpha}=\operatorname{Tr}\left(1-r_{\alpha}^{\dagger} r_{\alpha}\right)$, where the trace indicates again a summation over transport channels, is the spin-dependent dimensionless conductance of the ferromagnet, to be evaluated at the Fermi energy. The superscript (th) emphasizes that the fluctuations are caused by thermal agitation. The result for $S_{c, A B}^{(\text {th })}(\omega=0)$, where $B \neq A$, differs from the above expression only by a minus sign since current direction is defined as positive toward the ferromagnet on both sides, and charge current is conserved. The JohnsonNyquist noise [Eq. (21)] is a manifestation of the FDT since it relates the equilibrium current noise to the dissipation of energy parametrized by the conductance.

The thermal spin-current noise can be obtained in a similar way. At zero frequency

$$
S_{i j, A B}^{(\mathrm{th})}(0)=\frac{\hbar k_{B} T}{8 \pi} \sum_{\alpha \beta} \sigma_{i}^{\alpha \beta} \sigma_{j}^{\beta \alpha} \operatorname{Tr}\left[2 \delta_{A B}-s_{B A \alpha}^{\dagger} s_{B A \beta}-s_{A B \beta}^{\dagger} S_{A B \alpha}\right],
$$

where the scattering matrices should again be evaluated at the Fermi energy. The noise power of the $z$ component (polarized parallel to the magnetization) of the spin current,

$$
S_{z z, A A}^{(\text {th) }}=\frac{\hbar}{4 \pi} k_{B} T\left(g^{\uparrow}+g^{\downarrow}\right),
$$

differs from the charge current noise only by the squared conversion factor, $(\hbar / 2 e)^{2}$, from charge to spin currents. The transverse (polarized perpendicular to the magnetization) spin-current components fluctuate as

$$
S_{x x, A A}^{(\mathrm{th})}=S_{y y, A A}^{(\mathrm{th})}=\frac{\hbar}{4 \pi} k_{B} T\left(g_{A}^{\uparrow \downarrow}+g_{A}^{\downarrow \uparrow}\right) .
$$

The "spin-mixing" conductances $g_{L}^{\uparrow \downarrow}=\operatorname{Tr}\left[1-r_{\uparrow}\left(r_{\downarrow}\right)^{\dagger}\right]=\left(g_{L}^{\downarrow \uparrow}\right)^{*}$ and $g_{R}^{\uparrow \downarrow}=\operatorname{Tr}\left[1-r_{\uparrow}^{\prime}\left(r_{\downarrow}^{\prime}\right)^{\dagger}\right]=\left(g_{R}^{\downarrow \uparrow}\right)^{*}$ parametrize the absorbtivity of the ferromagnetic interfaces for transverse-polarized spin currents. We see that also the spin-current noise obeys the FDT since the spin-current correlators are proportional to the conductances for the respective spin-current components.

The cross correlation $S_{z z, L R}^{(\mathrm{th})}=-S_{z z, L L}^{(\mathrm{th})}$ reflects conservation of the longitudinal spin current in the ferromagnet since spin- flip scattering is disregarded. On the other hand, $S_{x x, L R}^{(\mathrm{th})}$ $=S_{y y, L R}^{(\text {th })}=0$ because the transverse spin current is absorbed at the interfaces to a ferromagnet thicker than the magnetic coherence length.

\section{Shot noise}

Shot noise of the electronic charge current is an out-ofequilibrium phenomenon proportional to the current bias. Shot noise is due to the discreteness of the electron charge, and the probabilistic incidence of electrons on the scatterer/ resistor. Let $\mu_{L}-\mu_{R}=e U$ with $U$ as the applied voltage, and take the temperature to be zero. We are here only concerned with the current fluctuations although in this case also the average charge current is nonzero. The average spin current accompanying the average charge current does not exert a torque on a single ferromagnet since the spin current is polarized along the direction of magnetization. From Eqs. (12), (13), and (16), and making use of the zero-temperature relations $f_{A}\left(1-f_{A}\right)=0$ and $\int d E\left(f_{L}-f_{R}\right)^{2}=e|U|$, we reproduce the well-known charge shot-noise expression ${ }^{6}$

$$
S_{c, A A}^{(\mathrm{sh})}(0)=\frac{e^{3}}{h}|U|\left[\operatorname{Tr}\left(r_{\uparrow}^{\dagger} r_{\uparrow} t_{\uparrow}^{\dagger} t_{\uparrow}\right)+\operatorname{Tr}\left(r_{\downarrow}^{\dagger} r_{\downarrow} t_{\downarrow}^{\dagger} t_{\downarrow}\right)\right] .
$$

Again, the scattering matrices should be evaluated at the Fermi energy, and the superscript (sh) emphasizes that this is shot noise. $S_{c, A B}^{(\mathrm{sh})}(0)=-S_{c, A A}^{(\mathrm{sh})}(0)$, where $B \neq A$. The spin shotnoise power is

$$
\begin{aligned}
S_{i j, A B}^{(\mathrm{sh})}(0)= & \frac{\hbar}{8 \pi} \sum_{\alpha \beta} \hat{\sigma}_{i}^{\alpha \beta} \hat{\sigma}_{j}^{\beta \alpha} \int d E \sum_{C D} f_{C}\left(1-f_{D}\right) \\
& \times \operatorname{Tr}\left[s_{A C \alpha}^{\dagger} s_{A D \beta} s_{B D \beta}^{\dagger} s_{B C \alpha}\right] .
\end{aligned}
$$

From this we find $S_{z z, L R}^{(\mathrm{sh})}=-S_{z z, L L}^{(\mathrm{sh})}$ and $S_{x x, L R}^{(\mathrm{th})}=S_{y y, L R}^{(\mathrm{th})}=0$, which hold for the same reasons as for the thermal noise.

\section{Magnetization noise and damping}

The absorption of fluctuating transverse spin currents at the ferromagnet's interfaces implies a fluctuating spintransfer torque on the magnetization. The resulting increment of the magnetization noise can be calculated using Eq. (4), which by conservation of angular momentum is modified by the spin torque $-\gamma_{0} \mathbf{I}_{s, \text { abs }}(t) /\left(M_{s} V\right)$. Here $\mathbf{I}_{s, \text { abs }}=\mathbf{I}_{s, L}+\mathbf{I}_{s, R}$ is the (instantaneously) absorbed spin current. (Recall that, on both sides of the ferromagnet, positive current direction is defined toward the magnet.) Since $\mathbf{I}_{s \text {,abs }}$ is perpendicular to $\mathbf{m}$, we may in general write $\mathbf{I}_{s, \text { abs }}=-\mathbf{m} \times\left[\mathbf{m} \times \mathbf{I}_{s \text {,abs }}\right]$, such that the modified stochastic LLG equation reads

$$
\begin{aligned}
\frac{d \mathbf{m}}{d t}= & -\gamma_{0} \mathbf{m} \times\left[\mathbf{H}_{\mathrm{eff}}+\mathbf{h}^{(0)}(t)\right] \\
& +\alpha_{0} \mathbf{m} \times \frac{d \mathbf{m}}{d t}+\frac{\gamma_{0}}{M_{s} V} \mathbf{m} \times\left[\mathbf{m} \times \mathbf{I}_{s_{\mathrm{abs}}}\right] .
\end{aligned}
$$

For the single ferromagnetic scatterer $\left\langle\mathbf{I}_{s, \text { abs }}\right\rangle=0$ but $\delta \mathbf{I}_{s, \text { abs }}(t) \neq 0$. We can thus define $\mathbf{h}(t)=-1 /\left(M_{s} V\right) \mathbf{m} \times \delta \mathbf{I}_{s}(t)$ to be a stochastic "magnetic" field that takes into account the (thermal or shot) spin-current noise that comes in addition to 
the intrinsic noise field $\mathbf{h}^{(0)}(t)$. The correlators of the field

$$
\left\langle h_{i}(t) h_{i}\left(t^{\prime}\right)\right\rangle=\frac{1}{M_{s}^{2} V^{2}} \sum_{A B} S_{j j, A B}\left(t-t^{\prime}\right),
$$

and

$$
\left\langle h_{i}(t) h_{j}\left(t^{\prime}\right)\right\rangle=-\frac{1}{M_{s}^{2} V^{2}} \sum_{A B} S_{j i, A B}\left(t-t^{\prime}\right),
$$

for $i, j=x, y ; i \neq j$ are directly obtained from the current noise. $\mathbf{h}(t)$ per definition has no component parallel to the magnetization. In the limit that the current noise is "white" on the relevant energy scales (temperature, applied voltage, and exchange splitting), we can approximate $S_{i j, A B}\left(t-t^{\prime}\right)$ $\approx S_{i j, A B}(\omega=0) \delta\left(t-t^{\prime}\right)$. Using Eq. (22) we then find the already advertised result [Eq. (10)]

$$
\left\langle h_{i}^{(\text {th) }}(t) h_{j}^{(\text {th })}\left(t^{\prime}\right)\right\rangle=2 k_{B} T \frac{\alpha^{\prime}}{\gamma_{0} M_{s} V} \delta_{i j} \delta\left(t-t^{\prime}\right),
$$

for the thermally (th) induced stochastic field. Here $\alpha^{\prime}$ $=\gamma_{0} \hbar \operatorname{Re}\left(g_{L}^{\uparrow \downarrow}+g_{R}^{\uparrow \downarrow}\right) /\left(4 \pi M_{s} V\right)$ is the spin-pumping enhancement of the Gilbert damping constant. This result is in agreement with the FDT [Eq. (3)] with a total Gilbert damping $\alpha=\alpha_{0}+\alpha^{\prime}$.

Using Eq. (26) and the unitarity of the scattering matrix we find for the stochastic field generated by the shot noise $\mathbf{h}^{\text {(sh): }}$

$$
\begin{aligned}
\left\langle h_{i}^{(\mathrm{sh})}(t) h_{j}^{(\mathrm{sh})}\left(t^{\prime}\right)\right\rangle= & \frac{\hbar}{4 \pi} \frac{e|U|}{M_{s}^{2} V^{2}} \delta_{i j} \delta\left(t-t^{\prime}\right)\left[\operatorname{Tr}\left(r_{\uparrow} r_{\uparrow}^{\dagger} t_{\downarrow}^{\prime} t_{\downarrow}^{\prime}\right)\right. \\
& \left.+\operatorname{Tr}\left(r_{\downarrow}^{\prime} r_{\downarrow}^{\dagger} t_{\uparrow}^{\dagger} t_{\uparrow}^{\dagger}\right)\right] .
\end{aligned}
$$

For a simple Stoner model it can be shown that, for typical experimental voltage drops in nanoscale metallic spin valves, $\mathbf{h}^{\text {(sh) }}$ can dominate $\mathbf{h}^{\text {(th) }}$ at temperatures of the order of 10 K. ${ }^{31}$ In the following section we concentrate on room temperature, at which shot noise may be disregarded.

\section{SPIN VALVES}

We now proceed to consider the noise properties of spinvalve nanopillars, i.e., layered structures consisting of two ferromagnets $F_{1}$ and $F_{2}$ with respective unit magnetization vectors $\mathbf{m}_{1}$ and $\mathbf{m}_{2}$ that are separated by a thin normal-metal spacer $N$, as sketched in Fig. 2. We first assume that $F_{2}$ is highly coercive, such that the fluctuations of it's magnetization vector are small. Such a "pinning" is routinely achieved in spin valves, e.g., by "exchange biasing." We relax this condition in Sec IV E.

The magnetization noise of the free layer $F_{1}$ is caused by intrinsic processes as well as by fluctuating spin currents in the neighboring normal metals. The latter source is affected by the presence of the second ferromagnet. Magnetoelectronic circuit theory ${ }^{38-40}$ enables us to compute the current fluctuations and thus the magnetization noise of composite structures such as spin valves.

Fluctuations of $\mathbf{m}_{1}$ cause an easily measurable electrical noise since the resistance of spin valves depends on the rela-

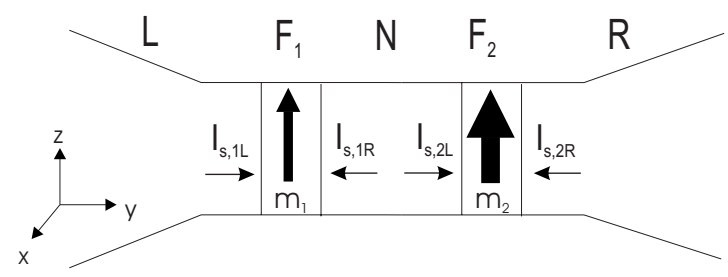

FIG. 2. A spin valve with two ferromagnets $F_{1}$ and $F_{2}$ with unit magnetization vectors $\mathbf{m}_{1}$ and $\mathbf{m}_{2}$ are here shown in the parallel (P) configuration $\mathbf{m}_{1}=\mathbf{m}_{2}=\mathbf{z}$. The magnetization of $F_{2}$ is fixed. The currents in the system are evaluated by magnetoelectronic circuit theory on the normal side of the interfaces, with positive directions defined by the arrows.

tive orientation of the magnetizations (GMR). Resistance noise is also interesting from a technological point of view since it affects the sensitivity of spin-valve read heads in magnetic storage devices.

In the following, we briefly explain the spin-current noise calculation by magnetoelectronic circuit theory. The stochastic field that acts on the free layer $F_{1}$ and the related Gilbert damping are found for different magnetic configurations. Using the LLG equation, we then calculate the fluctuations of the magnetization vector and the resulting resistance noise. We finish this section by considering spin valves in which both ferromagnets are identically susceptible to fluctuations.

\section{A. Circuit theory}

Magnetoelectronic circuit theory ${ }^{38-40}$ is a tool in determining transport properties of magnetoelectronic heterostructures such as the spin valve shown in Fig. 2. It is based on the division of a given structure into resistive elements (scatterers), nodes (low resistance interconnects), and reservoirs (voltage sources). The current through local resistors is calculated by LB scattering theory, which requires that nodes and reservoirs are characterized by (semiclassical) distribution functions. Here we take the ferromagnetic inserts as scatterers, the central normal-metal layer as a node, and the outer normal metals $L$ (left) and $R$ (right) as large reservoirs. The reservoirs are in thermal equilibrium, and hence characterized by Fermi-Dirac distribution functions $f_{L}=f\left(E-\mu_{L}\right)$ and $f_{R}=f\left(E-\mu_{R}\right)$, where $\mu_{L}$ and $\mu_{R}$ are the respective chemical potentials. Depending on the relative orientation of the magnetization vectors $\mathbf{m}_{1}$ and $\mathbf{m}_{2}$, there can be a nonequilibrium accumulation of spins on the normal-metal node, thus characterized by a scalar (charge) distribution function $f_{c N}$, and a vector spin distribution function $\mathbf{f}_{s N} . f_{c N}$ and $\mathbf{f}_{s N}$ form the distribution matrix $\hat{f}_{N}=\hat{1} f_{c N}+\hat{\boldsymbol{\sigma}} \cdot \mathbf{f}_{s N}$ in $2 \times 2$ spin space. As before, the ferromagnets are thicker than $\lambda_{c}$ but thin enough such that spin-flip processes can be disregarded. We also assume that spin flip in the central normal-metal node is negligible. We are in the diffuse scattering regime so $\hat{f}_{N}$ is isotropic and constant in space.

Referring back to Eq. (12), we now need quantum statistical averages $\left\langle a_{A m \alpha}^{\dagger}(E) a_{B n \beta}\left(E^{\prime}\right)\right\rangle=\delta_{A B} \delta_{m n} \delta\left(E-E^{\prime}\right) f_{A}^{\beta \alpha}(E)$, where $a_{B n \beta}$ is the annihilation operator for electrons moving in normal metal $A(A=L, R$, or $N)$ toward one of the ferromagnets, and $f_{A}^{\beta \alpha}$ is the $\beta \alpha$ component of the $2 \times 2$ semiclas- 
sical distribution matrix $\hat{f}_{A}$ in spin space. For the reservoirs $(A=L$ or $R)$, we simply have $f_{A}^{\beta \alpha}=\delta_{\beta \alpha} f\left(E-\mu_{A}\right)$. In contrast, in the central node the spin accumulation is not necessarily parallel to the spin-quantization axis in either of the ferromagnets, meaning that nondiagonal $(\beta \neq \alpha)$ terms in the distribution matrix do not vanish. The average charge current flowing from the right into ferromagnet $F_{1}$ can then be expressed by the generalized LB expressions ${ }^{38,40}$

$$
\begin{aligned}
\left\langle I_{c, 1 R}\right\rangle= & \frac{e}{h} \int d E\left[g_{1}^{\uparrow}\left(f_{c N}+\mathbf{f}_{s N} \cdot \mathbf{m}_{1}-f_{L}\right)\right. \\
& \left.+g_{1}^{\downarrow}\left(f_{c N}-\mathbf{f}_{s N} \cdot \mathbf{m}_{1}-f_{L}\right)\right],
\end{aligned}
$$

whereas the average spin current reads

$$
\begin{aligned}
\left\langle\mathbf{I}_{s, 1 R}\right\rangle= & \frac{1}{4 \pi} \int d E\left\{\mathbf { m } _ { 1 } \left[g_{1}^{\uparrow}\left(f_{c N}+\mathbf{f}_{s N} \cdot \mathbf{m}_{1}-f_{L}\right)\right.\right. \\
& \left.-g_{1}^{\downarrow}\left(f_{c N}-\mathbf{f}_{s N} \cdot \mathbf{m}_{1}-f_{L}\right)\right] \\
& \left.+2 \operatorname{Re} g_{1 R}^{\uparrow \downarrow} \mathbf{m}_{1} \times\left(\mathbf{f}_{S N} \times \mathbf{m}_{1}\right)+2 \operatorname{Im} g_{1 R}^{\uparrow \downarrow} \mathbf{f}_{s N} \times \mathbf{m}_{1}\right\} .
\end{aligned}
$$

Here $g_{1}^{\alpha}$ is the spin-dependent dimensionless conductance of $F_{1}$, and $g_{1}^{\uparrow \downarrow}$ is the mixing conductance of the interface between $F_{1}$ and the middle normal metal. The average charge current and the component of the spin current polarized along the magnetization are conserved through the ferromagnet. Hence $\left\langle I_{c, 1 L}\right\rangle=-\left\langle I_{c, 1 R}\right\rangle$ and $\left\langle\mathbf{I}_{s, 1 L}\right\rangle \cdot \mathbf{m}_{1}=-\left\langle\mathbf{I}_{s, 1 R}\right\rangle \cdot \mathbf{m}_{1}$. The transverse spin current is absorbed in the ferromagnet, leading to

$$
\begin{aligned}
\left\langle\mathbf{I}_{s, 1 L}\right\rangle= & \frac{1}{4 \pi} \int d E \mathbf{m}_{1}\left[g_{1}^{\uparrow}\left(f_{L}-f_{c N}-\mathbf{f}_{s N} \cdot \mathbf{m}_{1}\right)\right. \\
& \left.-g_{1}^{\downarrow}\left(f_{L}-f_{c N}+\mathbf{f}_{s N} \cdot \mathbf{m}_{1}\right)\right] .
\end{aligned}
$$

Similar expressions hold for the currents evaluated on the left and right sides of $F_{2}$. In order to keep the expressions simple we adopt from now on the parameters $g_{1}^{\alpha}=g_{2}^{\alpha}=g^{\alpha}$, and $g_{1 L}^{\uparrow \downarrow}=g_{1 R}^{\uparrow \downarrow}=g_{2 L}^{\uparrow \downarrow}=g_{2 R}^{\uparrow \downarrow}=g^{\uparrow \downarrow}$.

Since spin-flip processes are disregarded, both charge and spin are conserved on the middle normal-metal node:

$$
\begin{aligned}
& \left\langle I_{c, 1 R}\right\rangle+\left\langle I_{c, 2 L}\right\rangle=0, \\
& \left\langle\mathbf{I}_{s, 1 R}\right\rangle+\left\langle\mathbf{I}_{s, 2 L}\right\rangle=0 .
\end{aligned}
$$

Equations (31)-(35) come down to four equations for the four unknown components of the distribution matrix $\hat{f}_{N}$ as a function of the angle $\theta=\cos ^{-1}\left(\mathbf{m}_{1} \cdot \mathbf{m}_{2}\right)$ and the applied voltage $U=\left(\mu_{L}-\mu_{R}\right) / e$. Equation (31) then yields $\left\langle I_{c, 1 L}\right\rangle$ $=-\left\langle I_{c, 1 R}\right\rangle=\left\langle I_{c, 2 L}\right\rangle=-\left\langle I_{c, 2 R}\right\rangle \equiv I_{c}=G_{v} U$, where $^{38}$

$$
G_{v}=\frac{e^{2} g}{2 h}\left(1-P^{2} \frac{1-\cos \theta}{1-\cos \theta+\eta+\eta \cos \theta}\right)
$$

is the spin-valve conductance with material parameters $g$ $=g^{\uparrow}+g^{\downarrow}, P=\left(g^{\uparrow}-g^{\downarrow}\right) / g$, and $\eta=2 g^{\uparrow \downarrow} / g$.

\section{B. Current noise}

We combine spin and charge current fluctuations, e.g., $\Delta I_{c, 1 R}(t)$ and $\Delta \mathbf{I}_{s, 1 R}(t)$, respectively, on the right side of $F_{1}$, into a $2 \times 2$ matrix in spin space:

$$
\Delta \hat{I}_{1 R}(t)=\hat{1} \Delta I_{c, 1 R}(t)-(2 e / \hbar) \hat{\boldsymbol{\sigma}} \cdot \Delta \mathbf{I}_{s, 1 R}(t) .
$$

Since we focus on the zero-frequency noise, instantaneous charge and spin conservation in the central node may be assumed, i.e.,

$$
\Delta \hat{I}_{1 R}(t)+\Delta \hat{I}_{2 L}(t)=0
$$

which requires that the distribution matrix in the node fluctuates. The current fluctuations can then be written as

$$
\Delta \hat{I}_{1 R(2 L)}(t)=\delta \hat{I}_{1 R(2 L)}(t)+\frac{\partial\left\langle\hat{I}_{1 R(2 L)}\right\rangle}{\partial \hat{f}_{N}} \delta \hat{f}_{N}(t),
$$

where $\delta \hat{f}_{N}(t)$ are the fluctuations of the distribution matrix, and $\delta \hat{I}_{1 R(2 L)}(t)$ are the intrinsic fluctuations [when $\delta \hat{f}_{N}(t)=0$ ], coinciding with the fluctuations calculated for single ferromagnets in the previous section. Expression (39) applies also to the current fluctuations evaluated on the left side of ferromagnet $F_{1}$ and the right side of ferromagnet $F_{2}$. In the following, we focus on thermal current noise, recalling from Sec. IV D that, for typical voltage drops in spin valves, shot noise is only important at low temperatures.

From Eqs. (31), (32), (38), and (39), and results from Sec. III, we can evaluate the charge and spin-current fluctuations in the spin valve. The correlator $S_{c}(0)=\int d(t$ $\left.-t^{\prime}\right)\left\langle\Delta I_{c}(t) \Delta I_{c}\left(t^{\prime}\right)\right\rangle$ of the charge current fluctuations is simply related to conductance (36) by the following configuration-dependent FDT:

$$
S_{c}(\omega=0 ; \theta)=2 k_{B} T G_{v}(\theta) .
$$

In the low-frequency regime considered here, charge current noise is the same anywhere in the spin valve. $G_{v}$ can vary easily by a factor of two as a function of $\theta$, which corresponds to the same variation in noise power. Resistance noise via magnetization fluctuations is an additional source of electric noise that is treated below

The spin-current correlator $\left\langle\Delta I_{s_{i}, A}(t) \Delta I_{s_{i}, B}\left(t^{\prime}\right)\right\rangle$, where $i$ and $j$ denote Cartesian components and $A(B)=1 L, 1 R, 2 L$, or $2 R$, can be found analogously. Since spin current is not conserved at the ferromagnetic interfaces, the spin-current correlator depends on the location in the spin valve and is not directly observable. We therefore proceed to evaluate the magnetization fluctuations caused by the spin-current noise in the next subsection.

\section{Magnetization noise and damping}

The current-induced stochastic field acting on $F_{1}$ follows from the spin-current fluctuations as explained in Sec. III D. Here we discuss this field and, by using the FDT, the corresponding Gilbert damping enhancement in spin valves. In order to keep the algebra manageable, we focus on the most relevant parallel, antiparallel, and perpendicular configura- 
tions $(\cos \theta=0, \pm 1)$. The mixing conductances are taken to be identical for all four $F \mid N$ interfaces. In the semiclassical approach, intrinsic current fluctuations are not correlated across the node, implying that $\left\langle\delta I_{s_{i}, 1 R}(t) \delta I_{s_{j}, 2 L}\left(t^{\prime}\right)\right\rangle=0$.

\section{Parallel configuration}

For the parallel (P) magnetic configuration, $\mathbf{m}_{1} \cdot \mathbf{m}_{2}=1$, the thermal spin-current-induced stochastic magnetic field in ferromagnet $F_{1}$ reads

$$
\left\langle h_{i}^{(\text {th) }}(t) h_{j}^{(\text {th) }}\left(t^{\prime}\right)\right\rangle_{\mathrm{P}}=2 k_{B} T \frac{\alpha_{s v}}{\gamma_{0} M_{s} V} \delta_{i j} \delta\left(t-t^{\prime}\right),
$$

where $i, j$ label vector components perpendicular to the magnetization, and

$$
\alpha_{s v}=\frac{3 \gamma_{0} \hbar \operatorname{Re} g^{\uparrow \downarrow}}{8 \pi M_{s} V} .
$$

By the FDT, $\alpha_{s v}$ is identical to the spin-pumping enhancement of the Gilbert damping of the $F_{1}$ magnetization. This can be checked by following the steps outlined for a single ferromagnet, Eqs. (3)-(5). A possible exchange coupling between the ferromagnets modifies the dynamics via $\mathbf{H}_{\mathrm{eff}}$ in the LLG equation but does not affect the stochastic field and Gilbert damping.

The field correlator and damping for the parallel configuration is reduced by a factor of $3 / 4$ compared with Eq. (10) for the single ferromagnet sandwiched by normal metals. This result may be found also in a more direct way: using Eqs. (7) and (32) we can compute the net spin angular momentum leaving each of the ferromagnets when the magnetizations are slightly out of equilibrium, and by conservation of angular momentum, infer the corresponding enhancement of the Gilbert damping constant. The factor of 3/4 follows from the diffuse/chaotic nature of the node: half of the spin current that is pumped into the node is reflected back and reabsorbed by $F_{1}$.

One subtle point needs to be noted in this discussion: when the $F-N$ interfaces are nearly transparent, the interfacial conductance parameters from scattering theory should be corrected for spurious so-called Sharvin conductances (see Sec. IIB of Ref. 30). In practice, this will correct Eq. (42) only by a numerical prefactor close to one.

\section{Antiparallel configuration}

For the antiparallel (AP) configuration $\left(\mathbf{m}_{1} \cdot \mathbf{m}_{2}=-1\right)$,

$$
\left\langle h_{i}^{(\text {th })}(t) h_{j}^{(\text {th })}\left(t^{\prime}\right)\right\rangle_{\mathrm{AP}}=\left\langle h_{i}^{(\text {th })}(t) h_{j}^{(\text {th })}\left(t^{\prime}\right)\right\rangle_{\mathrm{P}},
$$

i.e., the current-induced noise and damping is the same as in the $\mathrm{P}$ configuration. This result holds only when the imaginary part of the mixing conductance is negligibly small.

\section{Perpendicular configuration}

When the $F_{2}$ magnetization is pinned along the $x$ direction and $\mathbf{m}_{1}$ points along the $z$ axis

$$
\left\langle h_{x}^{(\mathrm{th})}(t) h_{x}^{(\mathrm{th})}\left(t^{\prime}\right)\right\rangle_{\perp}=2 k_{B} T \frac{\alpha_{x x}^{\prime}}{\gamma_{0} M_{s} V} \delta\left(t-t^{\prime}\right),
$$

$$
\left\langle h_{y}^{(\mathrm{th})}(t) h_{y}^{(\mathrm{th})}\left(t^{\prime}\right)\right\rangle_{\perp}=2 k_{B} T \frac{\alpha_{y y}^{\prime}}{\gamma_{0} M_{s} V} \delta\left(t-t^{\prime}\right),
$$

where the subscript $\perp$ emphasizes that this is valid for the perpendicular configuration, and, according to the FDT,

$$
\alpha_{x x}^{\prime}=\frac{3 \gamma_{0} \hbar \operatorname{Re} g^{\uparrow \downarrow}}{8 \pi M_{s} V},
$$

$$
\alpha_{y y}^{\prime}=\frac{\gamma_{0} \hbar \operatorname{Re} g^{\uparrow \downarrow}}{4 \pi M_{s} V}\left[2-\frac{\eta\left(2-P^{2}+2 \eta\right)}{2(1+\eta)\left(1-P^{2}+\eta\right)}\right]
$$

is the spin-pumping-induced enhancement of the Gilbert damping. The cross correlators $\left\langle h_{x}^{\text {(th) }}(t) h_{y}^{(\text {th })}\left(t^{\prime}\right)\right\rangle_{\perp}$ $=\left\langle h_{y}^{\text {(th) }}(t) h_{x}^{\text {(th) }}\left(t^{\prime}\right)\right\rangle_{\perp}=0$. In noncollinear spin valves, the noise correlators and the Gilbert damping are therefore tensors. This can be accommodated by the LLG equation for $\mathbf{m}_{1}$ by a damping torque $\mathbf{m}_{1} \times \stackrel{\leftrightarrow}{\alpha} d \mathbf{m}_{1} / d t$, where the Gilbert damping tensor (in the plane perpendicular to the magnetization) reads

$$
\overleftrightarrow{\alpha}=\left(\begin{array}{cc}
\alpha_{0}+\alpha_{x x}^{\prime} & 0 \\
0 & \alpha_{0}+\alpha_{y y}^{\prime}
\end{array}\right)
$$

Note that the damping tensor must be written inside the cross product in the damping torque to ensure that the LLG equation preserves the length of the unit magnetization vector.

In our evaluation of the Gilbert damping [Eq. (46)], we have assumed that the outer left and right reservoirs have a fixed chemical potential which allows charge current fluctuations into the reservoirs. This is valid when the reservoirs are connected to external circuit elements with sufficiently long $R C$ times compared to the ferromagnetic resonance (FMR) precession period. In the opposite limit, when the reservoirs are fully decoupled from other circuit elements, charge current into the reservoirs must vanish at any time, and the chemical potentials fluctuate. This regime was considered in Ref. 49 with the result

$$
\begin{gathered}
\alpha_{x x}^{\prime}=\frac{3 \gamma_{0} \hbar \operatorname{Re} g^{\uparrow \downarrow}}{8 \pi M_{s} V}, \\
\alpha_{y y}^{\prime}=\frac{\gamma_{0} \hbar \operatorname{Re} g^{\uparrow \downarrow}}{4 \pi M_{s} V}\left[2-\frac{\eta}{1-P^{2}+\eta}\right] .
\end{gathered}
$$

\section{Resistance noise}

The fluctuations of the magnetization vector can be calculated by the LLG equation that incorporates the stochastic fields. Fluctuations in the magnetic configuration affect the electrical resistance that depends on the dot product $\mathbf{m}_{1} \cdot \mathbf{m}_{2}$. Resistance noise is an important issue for application of spinvalve read heads. ${ }^{43}$ Covington et al. $^{42}$ measured resistance noise in current-perpendicular-to-the-plane (CPP) spin valves, which are considered as an alternative for the conventional current-in-the-plane spin-valve read heads. We focus here on the zero-frequency resistance noise 


$$
S_{R}(\omega=0)=\int d\left(t-t^{\prime}\right)\left\langle\Delta R(t) \Delta R\left(t^{\prime}\right)\right\rangle,
$$

where $\Delta R(t)$ is the time-dependent deviation of the resistance from the time-averaged value.

Resistance noise can be measured, e.g., as voltage noise for constant current bias or as current noise for a constant voltage bias. The resistance noise comes on top of the Johnson-Nyquist noise discussed in Sec IV B and in Ref. 45. It has been shown to be a useful instrument to measure magnetization dynamics in tunnel junctions ("thermal FMR"). ${ }^{50}$ Current densities at which magnetization-induced noise start to dominate Johnson-Nyquist noise do not yet excite magnetization dynamics or correspond to appreciable shot-noise levels.

In the following, we derive the resistance noise in the parallel, antiparallel, and perpendicular configurations. Recall that the magnetization in ferromagnet $F_{2}$ is assumed pinned. The analysis of resistance noise in the case of two fluctuating magnetizations is left for the next section.

\section{Parallel configuration}

The total stochastic field in $F_{1}$ causes fluctuations $\delta \mathbf{m}_{1}(t)=\mathbf{m}_{1}(t)-\left\langle\mathbf{m}_{1}\right\rangle$ relative to its time-averaged equilibrium value $\left\langle\mathbf{m}_{1}\right\rangle$. For the parallel configuration $\left\langle\mathbf{m}_{1}\right\rangle=\mathbf{m}_{2}$, such that the dot product of the magnetizations is $\cos \theta$ $=\mathbf{m}_{1} \cdot \mathbf{m}_{2}=1-\delta \mathbf{m}_{1}^{2} / 2$, with $\theta$ as the angle between the magnetization directions. For small fluctuations we can expand the resistance to first order in $\delta \mathbf{m}_{1}^{2}$

$$
R\left(\mathbf{m}_{1} \cdot \mathbf{m}_{2}\right) \approx R(1)-\frac{1}{2} \delta \mathbf{m}_{1}^{2} \frac{\partial R(1)}{\partial \cos \theta}
$$

such that the resistance noise correlator becomes

$$
\begin{aligned}
\left\langle\Delta R(t) \Delta R\left(t^{\prime}\right)\right\rangle_{\mathrm{P}}= & \left\langle R(t) R\left(t^{\prime}\right)\right\rangle_{\mathrm{P}}-\langle R(t)\rangle_{\mathrm{P}}\left\langle R\left(t^{\prime}\right)\right\rangle_{\mathrm{P}} \\
= & \frac{1}{4}\left(\frac{\partial R(1)}{\partial \cos \theta}\right)^{2}\left[\left\langle\delta \mathbf{m}_{1}^{2}(t) \delta \mathbf{m}_{1}^{2}\left(t^{\prime}\right)\right\rangle_{\mathrm{P}}\right. \\
& \left.-\left\langle\delta \mathbf{m}_{1}^{2}(t)\right\rangle_{\mathrm{P}}\left\langle\delta \mathbf{m}_{1}^{2}\left(t^{\prime}\right)\right\rangle_{\mathrm{P}}\right]
\end{aligned}
$$

where the brackets denote statistical averaging around the parallel configuration. Assuming that the stochastic fields are Gaussian distributed, then so are the fluctuations of the magnetization vectors since the magnetization is a linear function of the stochastic fields. We may then employ Wick's theorem, ${ }^{51}$ according to which fourth-order moments of the fluctuations can be expressed in terms of the sum of products of second-order moments. We then arrive at

$$
\left\langle\Delta R(t) \Delta R\left(t^{\prime}\right)\right\rangle_{\mathrm{P}}=\frac{1}{2}\left(\frac{\partial R(1)}{\partial \cos \theta}\right)^{2} \sum_{i j}\left\langle\delta m_{1, i}(t) \delta m_{1, j}\left(t^{\prime}\right)\right\rangle_{\mathrm{P}}^{2},
$$

where $i$ and $j$ denote Cartesian components. From Eq. (36) we find

$$
\frac{\partial R(1)}{\partial \cos \theta}=-\frac{h P^{2}}{e^{2} g \eta} .
$$

Since the magnetization fluctuations are small, we may disregard their longitudinal component, whereas the correlator of the transverse fluctuations can be computed by the LLG equation.

We use the coordinate system in Fig. 2 with interfaces in the $x z$ plane. The LLG equation reads

$$
\frac{d \mathbf{m}_{1}}{d t}=-\gamma_{0} \mathbf{m}_{1} \times\left[\mathbf{H}_{\mathrm{eff}}+\mathbf{h}(t)\right]+\left(\alpha_{0}+\alpha_{s v}\right) \mathbf{m}_{1} \times \frac{d \mathbf{m}_{1}}{d t},
$$

where the total stochastic field $\mathbf{h}(t)=\mathbf{h}^{(0)}(t)+\mathbf{h}^{(\text {th })}(t)$ includes both the intrinsic field $\mathbf{h}^{(0)}(t)$ (see Sec. III) and the currentinduced field $\mathbf{h}^{(\text {th })}(t)$ from the previous section. $\alpha_{0}$ and $\alpha_{s v}$ are the corresponding Gilbert damping parameters. The effective field $\mathbf{H}_{\mathrm{eff}}=\mathbf{H}_{0}+\mathbf{H}_{a}+\mathbf{H}_{d}+\mathbf{H}_{e}$ contains the external field $\mathbf{H}_{0}$, the in-plane anisotropy field $\mathbf{H}_{a}$, the out-of-plane demagnetizing field $\mathbf{H}_{d}$, and the sum of dipolar and exchange fields $\mathbf{H}_{e}$. The external and anisotropy fields are both taken along the $z$ axis. We parametrize these fields by $\omega_{0}$ and $\omega_{a}$ as $\gamma \mathbf{H}_{0}=\omega_{0} \mathbf{z}$ and $\gamma \mathbf{H}_{a}=\omega_{a}\left(\mathbf{m}_{1} \cdot \mathbf{z}\right) \mathbf{z}$. The demagnetizing field is directed normal to the plane, i.e., along the $y$ axis, such that $\gamma \mathbf{H}_{d}=-\omega_{d}\left(\mathbf{m}_{1} \cdot \mathbf{y}\right) \mathbf{y}$ thereby introducing the parameter $\omega_{d}$. The dipolar and exchange couplings are described in terms of a Heisenberg coupling $-J \mathbf{m}_{1} \cdot \mathbf{m}_{2}$, which favors a parallel magnetic configuration for $J>0$ and an antiparallel one for $J<0$. This translates into the field $\gamma \mathbf{H}_{e}=\omega_{e} \mathbf{m}_{2}$, where $\omega_{e}$ $=\gamma J / M_{s} d$.

In the $\mathrm{P}$ configuration $\left\langle\mathbf{m}_{1}\right\rangle$ is aligned with the pinned $\mathbf{m}_{2}$ in the $+z$ direction, which can always be enforced by a sufficiently strong external field. Linearizing the LLG equation in the amplitude of the transverse fluctuations $\delta \mathbf{m}(t)$ $\approx \delta m_{x}(t) \mathbf{x}+\delta m_{y}(t) \mathbf{y}$, we find the magnetization noise correlator

$$
\left\langle\delta m_{i}(t) \delta m_{j}\left(t^{\prime}\right)\right\rangle_{\mathrm{P}}=\frac{\gamma_{0} k_{B} T \alpha}{\pi M_{S} V} \int d \omega e^{-i \omega\left(t-t^{\prime}\right)} U_{i j},
$$

by using the correlators of the stochastic fields. Here

$$
\begin{aligned}
& U_{x x}=\frac{\left[\omega^{2}+\left(\omega_{t}+\omega_{d}\right)^{2}\right]}{\left[\omega^{2}-\omega_{t}\left(\omega_{t}+\omega_{d}\right)\right]^{2}+\omega^{2} \alpha^{2}\left(2 \omega_{t}+\omega_{d}\right)^{2}}, \\
& U_{x y}=\frac{-i \omega\left(2 \omega_{t}+\omega_{d}\right)}{\left[\omega^{2}-\omega_{t}\left(\omega_{t}+\omega_{d}\right)\right]^{2}+\omega^{2} \alpha^{2}\left(2 \omega_{t}+\omega_{d}\right)^{2}}, \\
& U_{y y}=\frac{\left(\omega^{2}+\omega_{t}^{2}\right)}{\left[\omega^{2}-\omega_{t}\left(\omega_{t}+\omega_{d}\right)\right]^{2}+\omega^{2} \alpha^{2}\left(2 \omega_{t}+\omega_{d}\right)^{2}}, \\
& U_{y x}=-U_{x y},
\end{aligned}
$$

with $\alpha=\alpha_{0}+\alpha_{s v}$ and $\omega_{t}=\omega_{0}+\omega_{a}+\omega_{e}$. The above expressions hold for small damping, i.e., $\alpha_{0}^{2}, \alpha_{s v}^{2} \ll 1$. The zero-frequency resistance noise $S_{\mathrm{P}}(0)=\int d\left(t-t^{\prime}\right)\left\langle\Delta R(t) \Delta R\left(t^{\prime}\right)\right\rangle_{\mathrm{P}}$ is obtained by inserting Eq. (55) into Eq. (52): 


$$
S_{\mathrm{P}}(0)=\frac{1}{\pi}\left(\frac{h P^{2}}{e^{2} g \eta}\right)^{2}\left(\frac{\gamma_{0} k_{B} T \alpha}{M_{s} V}\right)^{2} \int d \omega\left(U_{x x}^{2}+U_{y y}^{2}-2 U_{x y}^{2}\right) .
$$

To gain insight into this rather complicated expression, it is convenient to make some simplifications. Although the demagnetizing field, which serves to stabilize the magnetization in the plane of the film, is important to get the right magnitude of the noise, we can gain physical understanding by disregarding it. Setting $\omega_{d}=0$, we find

$$
S_{\mathrm{P}}(0)=\left(\frac{\gamma_{0} k_{B} T}{M_{s} V}\right)^{2}\left(\frac{h P^{2}}{e^{2} g \eta}\right)^{2} \frac{1}{\omega_{t}^{3} \alpha} .
$$

Obviously, the resistance noise strongly depends on the parameter $\omega_{t}$. The external and anisotropy fields stabilize the magnetization, hence lowering the noise. The dipolar and exchange fields either stabilize or destabilize the magnetization, depending on the sign of the coupling constant $J$. We observe that the Gilbert damping also strongly affects the resistance noise. The resistance noise decreases with increasing damping because the suppression of the magnetic susceptibility by a large alpha turns out to be more important than the FDT-motivated increase in the stochastic field noise. Since $\alpha_{s v}$ can be of the same order as $\alpha_{0},{ }^{30}$ the importance of spin-current noise and spin pumping is evident.

When a constant voltage bias is applied, the resistance noise causes current noise. At sufficiently small bias, the Johnson-Nyquist current noise (Sec. IV B) always wins. However, at relatively high current densities, the effects of the resistance noise are very significant. That noise may be important for the next generation magnetoresistive spin valve read heads. ${ }^{43}$ For a quantitative comparison, which depends on many material parameters, it is important to use Eq. (60) and not Eq. (61) since the demagnetizing field has a large effect on the magnitude of the magnetization-induced noise. The magnetization-induced noise is most prominent for small structures since the ratio of Johnson-Nyquist noise to magnetization-induced noise scales with the volume of the ferromagnet.

\section{Antiparallel configuration}

When $J<0$, the dipolar and exchange couplings favor an AP configuration $\left(\left\langle\mathbf{m}_{1}\right\rangle=-\mathbf{m}_{2}\right)$ at zero external magnetic field. Following the recipe of the previous subsection, we find a resistance noise

$$
\left\langle\Delta R(t) \Delta R\left(t^{\prime}\right)\right\rangle_{\mathrm{AP}}=\frac{1}{2}\left(\frac{\partial R(-1)}{\partial \cos \theta}\right)^{2} \sum_{i j}\left\langle\delta m_{1, i}(t) \delta m_{1, j}\left(t^{\prime}\right)\right\rangle_{\mathrm{AP}}^{2},
$$

where the sensitivity of the resistance to the fluctuations is

$$
\frac{\partial R(-1)}{\partial \cos \theta}=-\frac{h P^{2} \eta}{e^{2} g\left(1-P^{2}\right)^{2}}
$$

Using the magnetization noise correlators from the linearized equation (54), the zero-frequency resistance noise becomes

$$
\begin{aligned}
S_{\mathrm{AP}}(0)= & \int d\left(t-t^{\prime}\right)\left\langle\Delta R(t) \Delta R\left(t^{\prime}\right)\right\rangle_{\mathrm{AP}} \\
= & \frac{1}{\pi}\left(\frac{h P^{2} \eta}{e^{2} g\left(1-P^{2}\right)^{2}}\right)^{2}\left(\frac{\gamma_{0} k_{B} T \alpha}{M_{s} V}\right)^{2} \\
& \times \int d \omega\left(V_{x x}^{2}+V_{y y}^{2}-2 V_{x y}^{2}\right),
\end{aligned}
$$

where $V_{i j}=U_{i j}\left(\omega_{t} \rightarrow \omega_{s}\right)$ with $\omega_{s}=\omega_{a}-\omega_{e}\left(\right.$ recall that $\left.\omega_{e}<0\right)$. Again disregarding the demagnetizing field strongly simplifies the expression:

$$
S_{\mathrm{AP}}(0)=\left(\frac{\gamma_{0} k_{B} T}{M_{s} V}\right)^{2}\left(\frac{h P^{2} \eta}{e^{2} g\left(1-P^{2}\right)^{2}}\right)^{2} \frac{1}{\omega_{s}^{3} \alpha} .
$$

As expected, the resistance noise decreases with increasing $\omega_{s}$. The anisotropy, dipolar, and exchange fields stabilize the magnetization, playing a role similar to that of the external field in the $\mathrm{P}$ configuration. The Gilbert damping enters in the same way as for the $\mathrm{P}$ configuration.

Except for the prefactor that reflects the sensitivity of the resistance to the magnetization fluctuations, $S_{\mathrm{P}}(0)$ and $S_{\mathrm{AP}}(0)$ are very similar. For the special case $\omega_{t}=\omega_{s}$,

$$
\frac{S_{\mathrm{P}}}{S_{\mathrm{AP}}}=\frac{\left(1-P^{2}\right)^{4}}{\eta^{4}}
$$

For, e.g., $P=0.7$ and $\eta=1$, this becomes $S_{\mathrm{P}} / S_{\mathrm{AP}} \approx 6 \%$ showing that the difference in noise level between the $\mathrm{P}$ and $\mathrm{AP}$ configurations can be substantial.

This asymmetry in the noise level between the P and AP configurations is consistent with the experimental results of Covington et al. on nearly cylindrical multilayer pillars. ${ }^{52}$ In these experiments the magnetizations were aligned parallel when the external magnetic field reached about 1500 Oe. Although we treat spin valves with two ferromagnetic films and Covington et al. dealt with multilayers of 4-15 magnetic films, it is likely that the difference between the noise properties of bilayers and multilayers is small, as the only local structural difference is the number of neighboring ferromagnets. This assertion is supported by the experiments of Covington et al. that did not reveal strong differences for nanopillars ranging from 4-15 layers.

\section{Perpendicular configuration}

We now investigate the perpendicular state $\left\langle\mathbf{m}_{1}\right\rangle \cdot \mathbf{m}_{2}=0$, assuming that $\mathbf{m}_{2}$ now has been pinned in the $x$ direction, whereas $\mathbf{m}_{1}$ is on average parallel to the $z$ axis, as before. In the following we assume that the interlayer exchange and dipolar couplings are negligibly small since otherwise the algebra and expressions become awkward.

Expanding the resistance to first order in the fluctuations $\delta \mathbf{m}_{1}$, we find in this case

$$
\left\langle\Delta R(t) \Delta R\left(t^{\prime}\right)\right\rangle_{\perp}=\left(\frac{\partial R(0)}{\partial \cos \theta}\right)^{2}\left\langle\delta m_{1 x}(t) \delta m_{1 x}\left(t^{\prime}\right)\right\rangle .
$$


The magnetization fluctuations affect the resistance noise in the perpendicular configuration to second order, unlike for the $\mathrm{P}$ and AP configurations, in which the leading term was of fourth order. The sensitivity of the resistance for this configuration is according to Eq. (36)

$$
\frac{\partial R(0)}{\partial \cos \theta}=-\frac{4 h P^{2} \eta}{e^{2} g\left(1+\eta-P^{2}\right)^{2}} .
$$

Linearizing Eq. (54) and using the correlators [Eqs. (44) and (45)] for the stochastic field, we find

$$
\left\langle\delta m_{1 x}(t) \delta m_{1 x}\left(t^{\prime}\right)\right\rangle=\frac{\gamma_{0} k_{B} T}{\pi M_{s} V} \int d \omega e^{-i \omega\left(t-t^{\prime}\right)} \frac{\omega^{2}\left(\alpha_{0}+\alpha_{y y}^{\prime}\right)+\left(\omega_{p}+\omega_{d}\right)^{2}\left(\alpha_{0}+\alpha_{x x}^{\prime}\right)}{\left[\omega^{2}-\omega_{p}\left(\omega_{p}+\omega_{d}\right)\right]^{2}+\omega^{2}\left[\omega_{p}\left(2 \alpha_{0}+\alpha_{x x}^{\prime}+\alpha_{y y}^{\prime}\right)+\omega_{d}\left(\alpha_{0}+\alpha_{x x}^{\prime}\right)\right]^{2}},
$$

where $\omega_{p}=\omega_{0}+\omega_{c}$. We then arrive at the zero-frequency resistance noise

$$
S_{\perp}(0)=\frac{2 \gamma_{0} k_{B} T}{M_{s} V}\left(\frac{4 h P^{2} \eta}{e^{2} g\left(1+\eta-P^{2}\right)^{2}}\right)^{2} \frac{\alpha_{0}+\alpha_{x x}^{\prime}}{\omega_{p}^{2}},
$$

quite different from that in the collinear configurations. In particular, the damping appears here in the numerator and there is no dependence on the demagnetizing field. Notice that since $S_{\perp}$ is quadratic in magnetic fluctuations [see Eq. (67)], it becomes linear in temperature, unlike $S_{\mathrm{P}}$ and $S_{\mathrm{AP}}$.

\section{E. Two identical ferromagnets}

We now investigate spin valves in which the ferromagnets are identical and hence equally susceptible to fluctuations, ${ }^{37}$ focusing now only on the $\mathrm{P}$ and AP configurations. The fluctuations of $F_{1}$ are $\delta \mathbf{m}_{1}(t)=\mathbf{m}_{1}(t)-\left\langle\mathbf{m}_{1}\right\rangle$ and those of $F_{2}$ are $\delta \mathbf{m}_{2}(t)=\mathbf{m}_{2}(t)-\left\langle\mathbf{m}_{2}\right\rangle$. As before, we choose the $z$ axis so that the time-averaged equilibrium values are $\left\langle\mathbf{m}_{1}\right\rangle=\left\langle\mathbf{m}_{2}\right\rangle=\mathbf{z}$ for the parallel configuration, and $\left\langle\mathbf{m}_{1}\right\rangle=-\left\langle\mathbf{m}_{2}\right\rangle=\mathbf{z}$ for the antiparallel. The dot product of the magnetizations is $\mathbf{m}_{1} \cdot \mathbf{m}_{2}$ $= \pm 1 \mp\left(\delta \mathbf{m}^{\bar{\gamma}}\right)^{2} / 2$, where the upper (lower) sign holds for the $\mathrm{P}(\mathrm{AP})$ orientation and $\delta \mathbf{m}^{\mp}=\delta \mathbf{m}_{1} \mp \delta \mathbf{m}_{2}$. For small fluctuations, we can expand the resistance to first order in $\left(\delta \mathbf{m}^{\mp}\right)^{2}$, finding

$$
R\left(\mathbf{m}_{1} \cdot \mathbf{m}_{2}\right) \approx R( \pm) \mp \frac{1}{2}\left(\delta \mathbf{m}^{\overline{ }}\right)^{2} \frac{\partial R( \pm 1)}{\partial \cos \theta} .
$$

The resistance noise is then

$$
\begin{aligned}
\left\langle\Delta R(t) \Delta R\left(t^{\prime}\right)\right\rangle_{\mathrm{P} / \mathrm{AP}}= & \left\langle R(t) R\left(t^{\prime}\right)\right\rangle_{\mathrm{P} / \mathrm{AP}}-\langle R(t)\rangle_{\mathrm{P} / \mathrm{AP}}\left\langle R\left(t^{\prime}\right)\right\rangle_{\mathrm{P} / \mathrm{AP}} \\
= & \frac{1}{4}\left(\frac{\partial R( \pm 1)}{\partial \cos \theta}\right)^{2}\left[\left\langle\left(\delta \mathbf{m}^{\mp}\right)^{2}\left(\delta \mathbf{m}^{\mp}\right)^{2}\right\rangle_{\mathrm{P} / \mathrm{AP}}\right. \\
& \left.-\left\langle\left(\delta \mathbf{m}^{\mp}\right)^{2}\right\rangle_{\mathrm{P} / \mathrm{AP}}\left\langle\left(\delta \mathbf{m}^{\mp}\right)^{2}\right\rangle_{\mathrm{P} / \mathrm{AP}}\right]
\end{aligned}
$$

which by employing Wick's theorem becomes

$$
\left\langle\Delta R(t) \Delta R\left(t^{\prime}\right)\right\rangle_{\mathrm{P} / \mathrm{AP}}=\frac{1}{2}\left(\frac{\partial R( \pm 1)}{\partial \cos \theta}\right)^{2} \sum_{i j}\left\langle\delta m_{i}^{\mp}(t) \delta m_{j}^{\mp}\left(t^{\prime}\right)\right\rangle_{\mathrm{P} / \mathrm{AP}}^{2}
$$

Letting the subscripts $k$ and $l$ refer to ferromagnet 1 or 2 , the LLG equation in this case reads

$$
\begin{aligned}
\frac{d \mathbf{m}_{k}}{d t}= & -\gamma_{0} \mathbf{m}_{k} \times\left[\mathbf{H}_{\mathrm{eff}}+\mathbf{h}_{k}(t)\right]+\left(\alpha_{0}+\alpha_{s v}\right) \mathbf{m}_{k} \times \frac{d \mathbf{m}_{k}}{d t} \\
& +\frac{\alpha_{s v}}{3} \mathbf{m}_{l} \times \frac{d \mathbf{m}_{l}}{d t},
\end{aligned}
$$

where the effective field $\mathbf{H}_{\text {eff }}$ is now taken to be equal for both ferromagnets. Due to current conservation, the ferromagnets' respective current-induced stochastic fields are not independent of each other. With the spin-current noise calculated in Sec. IV B, and following the recipe in Sec. III D, we find

$$
\left\langle h_{1, i}^{\text {(th) }}(t) h_{2, j}^{(\text {th })}\left(t^{\prime}\right)\right\rangle_{\mathrm{P}}=-2 k_{B} T \frac{\alpha_{s v} / 3}{\gamma_{0} M_{s} V} \delta_{i j} \delta\left(t-t^{\prime}\right)
$$

for the $\mathrm{P}$ configuration, and

$$
\left\langle h_{1, i}^{\text {(th) }}(t) h_{2, j}^{(\mathrm{th})}\left(t^{\prime}\right)\right\rangle_{\mathrm{AP}}=2 k_{B} T \frac{\alpha_{s v} / 3}{\gamma_{0} M_{s} V} \delta_{i j} \delta\left(t-t^{\prime}\right)
$$

for the AP configuration (as before $i, j$ label components perpendicular to the magnetization direction). $\alpha_{s v}$ is defined in Eq. (42). Naturally, the bulk fields $\mathbf{h}_{1}^{(0)}$ and $\mathbf{h}_{2}^{(0)}$ are uncorrelated. The last term in the LLG [Eq. (74)] represent the dynamic spin-exchange coupling: ${ }^{30,53}$ It is the spin current pumped from ferromagnet $l$ (see Sec. III) that is transmitted to and subsequently absorbed by ferromagnet $k$. Since the normal-metal node is chaotic, this amounts to one third of the net total spin current pumped out of ferromagnet $l$. This dynamic coupling was not present in spin valves in which one magnetization is not moving at all.

By linearizing Eq. (74) in $\delta \mathbf{m}_{k}(t)$ we can evaluate the desired magnetization noise correlators that are to be inserted in Eq. (73). The zero-frequency resistance noise for the P and AP configurations then, respectively, reads

$$
S_{\mathrm{P}}(0)=\frac{1}{\pi}\left(\frac{h P^{2}}{e^{2} g \eta}\right)^{2}\left(\frac{2 \gamma_{0} k_{B} T}{M_{s} V}\right)^{2} \int d \omega\left(Z_{x x}^{2}+Z_{y y}^{2}-2 Z_{x y}^{2}\right),
$$

and 


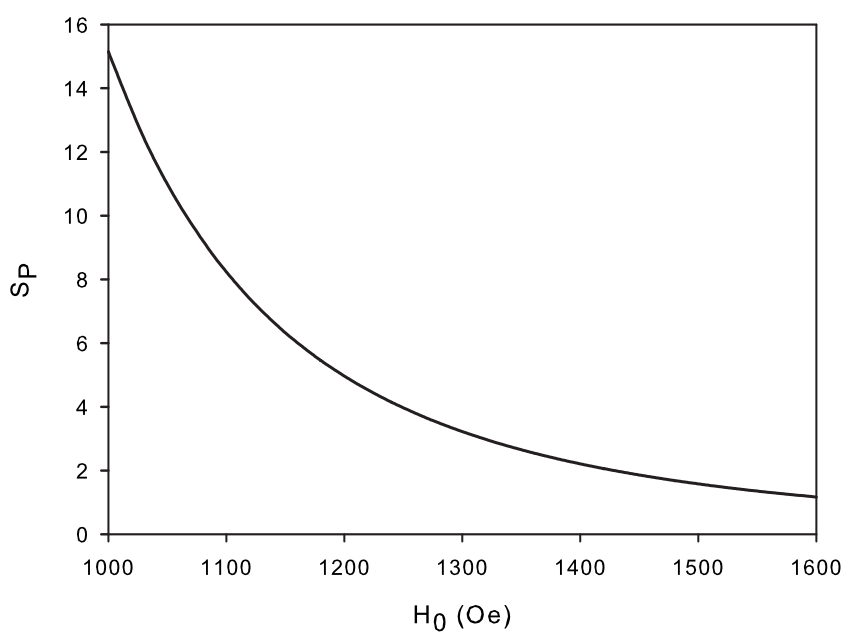

FIG. 3. The resistance noise in the $\mathrm{P}$ configuration as a function of the externally applied magnetic field, given in units of $\left(10^{-7} / \pi\right)\left(h P^{2} / e^{2} g \eta\right)^{2}\left(2 \gamma_{0} k_{B} T / M_{s} V\right)^{2}$. The parameters used are $\alpha_{0}$ $=\alpha_{s v}=0.01, \omega_{c} / \gamma_{0}=\omega_{d} / \gamma_{0}=100 \mathrm{Oe}$, and $J=-0.10 \mathrm{erg} / \mathrm{cm}^{2}$.

$$
S_{\mathrm{AP}}(0)=\frac{1}{\pi}\left(\frac{h P^{2} \eta}{e^{2} g\left(1-P^{2}\right)^{2}}\right)^{2}\left(\frac{2 \gamma_{0} k_{B} T}{M_{s} V}\right)^{2} \int d \omega\left(X_{x}^{2}+X_{y}^{2}\right)
$$

Here

$$
Z_{x x}=\frac{\alpha_{t}\left[\omega^{2}+\left(\omega_{i}+\omega_{d}\right)^{2}\right]}{\left[\omega^{2}-\omega_{i}\left(\omega_{i}+\omega_{d}\right)\right]^{2}+\omega^{2} \alpha_{t}^{2}\left(2 \omega_{i}+\omega_{d}\right)^{2}},
$$

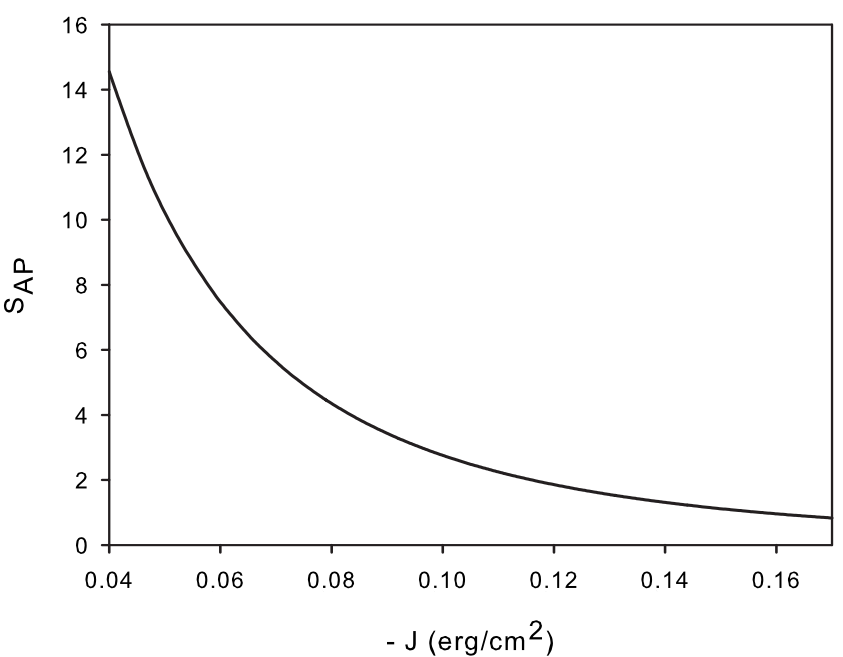

FIG. 4. The resistance noise in the AP configuration as a function of the dipolar and exchange couplings between the ferromagnets, given in units of $\left(10^{-7} / \pi\right)\left[h P^{2} \eta / e^{2} g(1\right.$ $\left.\left.-P^{2}\right)^{2}\right]^{2}\left(2 \gamma_{0} k_{B} T / M_{s} V\right)^{2}$. The parameters used are $\alpha_{0}=\alpha_{s v}=0.01$, $\omega_{c} / \gamma_{0}=\omega_{d} / \gamma_{0}=100$ Oe, and $\mathbf{H}_{0}=0$.

$$
Z_{x y}=\frac{-i \omega \alpha_{t}\left(2 \omega_{i}+\omega_{d}\right)}{\left[\omega^{2}-\omega_{i}\left(\omega_{i}+\omega_{d}\right)\right]^{2}+\omega^{2} \alpha_{t}^{2}\left(2 \omega_{i}+\omega_{d}\right)^{2}},
$$

$$
Z_{y y}=\frac{\alpha_{t}\left(\omega^{2}+\omega_{i}^{2}\right)}{\left[\omega^{2}-\omega_{i}\left(\omega_{i}+\omega_{d}\right)\right]^{2}+\omega^{2} \alpha_{t}^{2}\left(2 \omega_{i}+\omega_{d}\right)^{2}},
$$

and

$$
\begin{aligned}
& X_{x}=\frac{\omega^{2} \alpha_{s}+\left(\omega_{c}+\omega_{d}\right)^{2} \alpha_{t}}{\left[\omega^{2}+\left(\omega_{c}+\omega_{d}\right)\left(2 \omega_{e}-\omega_{c}\right)\right]^{2}+\omega^{2}\left(2 \omega_{x} \alpha_{s}-2 \omega_{c} \alpha-\omega_{d} \alpha_{t}\right)^{2}}, \\
& X_{y}=\frac{\omega^{2} \alpha_{s}+\omega_{c}^{2} \alpha_{t}}{\left[\omega^{2}+\omega_{c}\left(2 \omega_{e}-\omega_{c}-\omega_{d}\right)\right]^{2}+\omega^{2}\left(2 \omega_{x} \alpha_{s}-2 \omega_{c} \alpha-\omega_{d} \alpha_{s}\right)^{2}} .
\end{aligned}
$$

For convenience, we defined $\alpha_{s}=\alpha_{0}+2 \alpha_{s v} / 3, \quad \alpha_{t}=\alpha_{0}$ $+4 \alpha_{s v} / 3, \alpha=\alpha_{0}+\alpha_{s v}$ (note the difference between $\alpha, \alpha_{s}$, and $\left.\alpha_{t}\right)$, and $\omega_{i}=\omega_{0}+\omega_{a}+2 \omega_{x}$. The above expressions hold for small damping, i.e., $\alpha_{0}^{2}, \alpha_{s v}^{2} \ll 1$.

Compared to the results in the previous section, we see that Eq. (77) is similar to Eq. (60), whereas Eq. (78) differs considerably from Eq. (64). This is due to the static dipolar and exchange couplings, and the dynamic spin-exchange coupling, whose effects on the noise are modified by the presence of the second fluctuating ferromagnet. In particular, the latter coupling causes the Gilbert damping constant to enter Eqs. (77) and (78) differently. Equation (77) decreases with the external field, and Eq. (78) decreases with the dipolar and exchange couplings, as expected, and as shown in
Figs. 3 and 4. The noise level is in general higher when both ferromagnets fluctuate than when only one does.

The resistance noise is governed by a number of material parameters. Depending on these parameters, the noise level in the $\mathrm{P}$ configuration can differ substantially from that in the AP configuration. Note that Eq. (78) reduces to that of Ref. 37 when the demagnetizing field is disregarded, i.e., when $\omega_{d} \rightarrow 0$, whereas Eq. (77) does when $\omega_{a} \rightarrow 0$ and $\omega_{d} \rightarrow-\omega_{a}$ since the external field in our earlier work was perpendicular to the anisotropy field. The considerable difference between $S_{\mathrm{P}}(0)$ and $S_{\mathrm{AP}}(0)$ in typical experimental spin-valve setups can partly be explained by the dynamic exchange coupling. ${ }^{37}$ However, also the sensitivity of the resistance to magnetic configuration changes can be important, as shown in the pre- 
vious section. The demagnetizing field also significantly affects the numerical result for the noise level since it stabilizes the magnetization, in both the $\mathrm{P}$ and $\mathrm{AP}$ configurations.

\section{CONCLUSIONS}

In magnetoelectronic circuits, there are coupled current and magnetization fluctuations associated with dissipation and out-of-equilibrium properties. Current and magnetization dynamics interact via the reciprocal effects of spin-transfer torque and spin pumping affecting not only the time averaged but also the noise properties. Via the fluctuationdissipation theorem this is also manifested as a cross-talk of current and magnetization dissipations. Using scattering and magnetoelectronic circuit theories, we explicitly demonstrate the effect of spin-current fluctuations on the magnetization in ferromagnetic multilayers. Via a fluctuating spin-transfer torque, the current noise causes enhanced magnetization noise. Both thermal equilibrium (Johnson-Nyquist) current noise and nonequilibrium shot noise are taken into account. Taking advantage of the fluctuation-dissipation theorem, we relate the equilibrium electric (current and voltage) and magnetic (magnetization and field) noises to the corresponding dissipation of energy.

In spin valves, in which one of the ferromagnets is pinned, magnetization noise and damping associated with the free layers are tensors that depend on the magnetic configuration. The magnetization fluctuations in spin valves induce resistance noise, which is identified as a prominent source of electric noise at relatively high current densities, and is experimentally detectable. The resistance noise is shown to vary considerably with the magnetic configuration, partly due to the angular dependence of the GMR. The contribution from spin-current noise to resistance noise is considerable, and may be an issue for the next generation magnetoresistive spin-valve read heads and memory applications.

\section{ACKNOWLEDGMENTS}

We thank Mark Covington for sharing his results prior to publication as well as Neil Smith and Hans Joakim Skadsem for discussions. This work was supported by EC Contract No. IST-033749 "DynaMax.”
${ }^{1}$ T. Kawahara et al., IEEE J. Solid-State Circuits 43, 109 (2008).

${ }^{2}$ J. B. Johnson, Phys. Rev. 32, 97 (1928).

${ }^{3}$ H. Nyquist, Phys. Rev. 32, 110 (1928).

${ }^{4}$ H. B. Callen and T. A. Welton, Phys. Rev. 83, 34 (1951).

${ }^{5}$ L. D. Landau, E. M. Lifshitz, and L. P. Pitaevskii, Statistical Physics, Part 1, 3rd ed. (Pergamon Press, New York, 1980).

${ }^{6}$ Y. M. Blanter and M. Büttiker, Phys. Rep. 336, 1 (2000).

${ }^{7}$ W. Belzig and M. Zareyan, Phys. Rev. B 69, 140407(R) (2004).

${ }^{8}$ B. R. Bulka, J. Martinek, G. Michalek, and J. Barnaś, Phys. Rev. B 60, 12246 (1999).

${ }^{9}$ A. Lamacraft, Phys. Rev. B 69, 081301(R) (2004).

${ }^{10}$ E. G. Mishchenko, Phys. Rev. B 68, 100409(R) (2003).

${ }^{11}$ Y. Tserkovnyak and A. Brataas, Phys. Rev. B 64, 214402 (2001).

${ }^{12}$ M. Zareyan and W. Belzig, Phys. Rev. B 71, 184403 (2005).

${ }^{13}$ W. F. Brown, Phys. Rev. 130, 1677 (1963).

${ }^{14}$ V. L. Safonov and H. N. Bertram, Phys. Rev. B 71, 224402 (2005).

${ }^{15}$ N. Smith, J. Appl. Phys. 92, 3877 (2002).

${ }^{16}$ R. H. Koch, G. Grinstein, G. A. Keefe, Y. Lu, P. L. Trouilloud, W. J. Gallagher, and S. S. P. Parkin, Phys. Rev. Lett. 84, 5419 (2000).

${ }^{17}$ Z. Li and S. Zhang, Phys. Rev. B 69, 134416 (2004).

${ }^{18}$ E. B. Myers, F. J. Albert, J. C. Sankey, E. Bonet, R. A. Buhrman, and D. C. Ralph, Phys. Rev. Lett. 89, 196801 (2002).

${ }^{19}$ W. Wernsdorfer, E. B. Orozco, K. Hasselbach, A. Benoit, B. Barbara, N. Demoncy, A. Loiseau, H. Pascard, and D. Mailly, Phys. Rev. Lett. 78, 1791 (1997).

${ }^{20}$ T. L. Gilbert, Phys. Rev. 100, 1243 (1955) [Abstract only; full report, Armor Research Foundation Project No. A059, Supplementary Report, May 1, 1956 (unpublished).

${ }^{21}$ T. L. Gilbert, IEEE Trans. Magn. 40, 3443 (2004).

${ }^{22}$ S. M. Bhagat and P. Lubitz, Phys. Rev. B 10, 179 (1974).

${ }^{23}$ K. Gilmore, Y. U. Idzerda, and M. D. Stiles, Phys. Rev. Lett. 99,
027204 (2007)

${ }^{24}$ A. Brataas, Y. Tserkovnyak, and G. E. W. Bauer, Phys. Rev. Lett. 101, 037207 (2008).

${ }^{25}$ H. Kohno, G. Tatara, and J. Shibata, J. Phys. Soc. Jpn. 75, 113706 (2006).

${ }^{26}$ V. Korenman and R. E. Prange, Phys. Rev. B 6, 2769 (1972).

${ }^{27}$ H. J. Skadsem, Y. Tserkovnyak, A. Brataas, and G. E. W. Bauer, Phys. Rev. B 75, 094416 (2007).

${ }^{28}$ H. Suhl, IEEE Trans. Magn. 34, 1834 (1998).

${ }^{29}$ Y. Tserkovnyak, A. Brataas, and G. E. W. Bauer, Phys. Rev. Lett. 88, 117601 (2002).

${ }^{30}$ Y. Tserkovnyak, A. Brataas, G. E. W. Bauer, and B. I. Halperin, Rev. Mod. Phys. 77, 1375 (2005).

${ }^{31}$ J. Foros, A. Brataas, Y. Tserkovnyak, and G. E. W. Bauer, Phys. Rev. Lett. 95, 016601 (2005).

${ }^{32}$ A. Rebei and M. Simionato, Phys. Rev. B 71, 174415 (2005).

${ }^{33}$ L. Berger, Phys. Rev. B 54, 9353 (1996).

${ }^{34}$ J. A. Katine, F. J. Albert, R. A. Buhrman, E. B. Myers, and D. C. Ralph, Phys. Rev. Lett. 84, 3149 (2000).

${ }^{35}$ E. B. Myers, D. C. Ralph, J. A. Katine, R. N. Louie, and R. A. Buhrman, Science 285, 867 (1999).

${ }^{36}$ J. C. Slonczewski, J. Magn. Magn. Mater. 159, L1 (1996).

${ }^{37}$ J. Foros, A. Brataas, G. E. W. Bauer, and Y. Tserkovnyak, Phys. Rev. B 75, 092405 (2007).

${ }^{38}$ A. Brataas, G. E. W. Bauer, and P. J. Kelly, Phys. Rep. 427, 157 (2006).

${ }^{39}$ A. Brataas, Y. V. Nazarov, and G. E. W. Bauer, Phys. Rev. Lett. 84, 2481 (2000).

${ }^{40}$ A. Brataas, Y. V. Nazarov, and G. E. W. Bauer, Eur. Phys. J. B 22, 99 (2001).

${ }^{41}$ J. Foros, A. Brataas, Y. Tserkovnyak, and G. E. W. Bauer, Phys. Rev. B 78, 140402(R) (2008).

${ }^{42}$ M. Covington, M. AlHajDarwish, Y. Ding, N. J. Gokemeijer, and 
M. A. Seigler, Phys. Rev. B 69, 184406 (2004).

${ }^{43}$ N. Smith and P. Arnett, Appl. Phys. Lett. 78, 1448 (2001).

${ }^{44}$ J. Foros, Ph.D. thesis, NTNU Trondheim, 2008.

${ }^{45}$ Jiang Xiao, Gerrit E. W. Bauer, Sadamichi Maekawa, and Arne Brataas, Phys. Rev. B 79, 174415 (2009).

${ }^{46}$ L. D. Landau, E. M. Lifshitz, and L. P. Pitaevskii, Statistical Physics, Part 2, 2nd ed. (Pergamon Press, New York, 1980).

${ }^{47}$ Y. Tserkovnyak and M. Mecklenburg, Phys. Rev. B 77, 134407 (2008).

${ }^{48}$ M. Büttiker, Phys. Rev. B 46, 12485 (1992).

${ }^{49}$ Y. Tserkovnyak, A. Brataas, and G. E. W. Bauer, Phys. Rev. B
67, 140404(R) (2003).

${ }^{50}$ A. M. Deac, A. Fukushima, H. Kubota, H. Maehara, Y. Suzuki, S. Yuasa, Y. Nagamine, K. Tsunekawa, D. D. Djayaprawira, and N. Watanabe, Nat. Phys. 4, 803 (2008).

${ }^{51}$ K. Triantafyllopoulos, Mathematical Scientist 28, 125 (2003).

${ }^{52}$ M. Covington, Y. Yang, T. M. Crawford, N. J. Gokemeijer, M. A. Seigler, and M. K. Minor, Digest of The Magnetic Recording Conference (TMRC), 2005, Stanford, CA (unpublished).

${ }^{53}$ B. Heinrich, Y. Tserkovnyak, G. Woltersdorf, A. Brataas, R. Urban, and G. E. W. Bauer, Phys. Rev. Lett. 90, 187601 (2003). 